\title{
A general catalogue of molecular hydrogen emission-line objects (MHOs) in outflows from young stars ${ }^{\star \star \star}$
}

\author{
C. J. Davis ${ }^{1}$, R. Gell ${ }^{1,2}$, T. Khanzadyan ${ }^{3}$, M. D. Smith ${ }^{4}$, and T. Jenness ${ }^{1}$ \\ 1 Joint Astronomy Centre, 660 North A'ohōkū Place, University Park, Hilo, Hawaii 96720, USA \\ e-mail: c.davis@jach.hawaii.edu \\ 2 Faculty of Engineering, University of Victoria, Victoria BC, V8W 3P6, Canada \\ 3 Centre for Astronomy, Department of Experimental Physics, National University of Ireland, Galway, Ireland \\ ${ }^{4}$ Centre for Astrophysics \& Planetary Science, School of Physical Sciences, University of Kent, Canterbury CT2 7NR, UK
}

Received 28 October 2009 / Accepted 15 December 2009

\section{ABSTRACT}

\begin{abstract}
We present a catalogue of Molecular Hydrogen emission-line Objects (MHOs) in outflows from young stars, most of which are deeply embedded. All objects are identified in the near-infrared lines of molecular hydrogen, all reside in the Milky Way, and all are associated with jets or molecular outflows. Objects in both low and high-mass star forming regions are included. This catalogue complements the existing database of Herbig-Haro objects; indeed, for completeness, $\mathrm{HH}$ objects that are detected in $\mathrm{H}_{2}$ emission are included in the MHO catalogue.
\end{abstract}

Key words. catalogs - stars: formation - Herbig-Haro objects - infrared: ISM - ISM: jets and outflows

\section{Introduction}

For over 30 years, astronomers have been observing HerbigHaro $(\mathrm{HH})$ objects, jets and outflows in star forming regions in the near-infrared. The molecular hydrogen $v=1-0 \mathrm{~S}(1)$ line at $2.122 \mu \mathrm{m}$ is a particularly powerful tracer of shock-excited features in molecular outflows (e.g. Wilking et al. 1990; Garden et al. 1990; Zealey et al. 1992; Gredel 1994; Davis \& Eislöffel 1995; Zinnecker, et al. 1998; Reipurth et al. 1999; Eislöffel 2000; Stanke et al. 2002; Caratti o Garatti et al. 2006; Walawender et al. 2009; Davis et al. 2009). Although excited in a similar way to $\mathrm{HH}$ objects, these molecular hydrogen emission-line features are often too deeply embedded to be seen at optical wavelengths. They are thus not classified as HH objects, which are strictly defined by optical criteria, and are instead labelled in a rather hap-hazard way, often with the authors' initials. In large on-line databases this can lead to some ambiguity.

Our goal with this catalogue was therefore to develop a selfconsistent list of $\mathrm{H}_{2}$ emission-line objects, in a manner similar to that used so successfully for $\mathrm{HH}$ objects. With guidance from the International Astronomical Union (IAU) Working Group on Designations, we have adopted a scheme that simply lists objects sequentially, although objects are grouped by region (see below). The simple acronym "MHO", for molecular hydrogen emissionline object, is used to refer to these objects. This acronym has been approved by the IAU registry, and has been entered into the on-line Reference Dictionary of Nomenclature of Celestial Objects $^{1}$.

\footnotetext{
* http://www.jach.hawaii.edu/UKIRT/MHCat/
}

$\star \star$ Tables A.1 to A.15 are also available in electronic form at the CDS via anonymous ftp to cdsarc.u-strasbg. fr (130.79.128.5) or via

http://cdsweb.u-strasbg.fr/cgi-bin/qcat?]/A+A/511/A24

${ }^{1}$ http://cdsweb.u-strasbg.fr/cgi-bin/Dic?MHO

\section{What constitutes an MHO}

Only objects associated with outflows from young stellar objects (YSOs) and protostars are included in this catalogue. We do not list outflows from evolved stars (AGB stars or proto-planetary nebulae) or extra-galactic sources. Also, objects should be spatially resolved; unresolved emission-line regions associated with an accretion disk or the base of an outflow (that were observed spectroscopically) are not listed.

Since large-scale imaging surveys are now revealing tens or even hundreds of objects in some regions (e.g. Stanke et al. 2002; Khanzadyan et al. 2004b; Walawender et al. 2009; Davis et al. 2009), spectroscopic confirmation of every feature is not usually practical (although multi-object spectrographs that operate in the infrared will certainly help in this regard). Therefore, to properly identify an MHO, narrow-band molecular hydrogen images should be accompanied by either adjacent narrowband continuum images or (flux-scaled) broad-band $\mathrm{K}$ images. It is obviously important that these shock-excited features be distinguished from wisps and knots of continuum emission. Morphology alone should not be used to identify MHOs, although the shape of an object may help distinguish features in outflows from fluorescently excited emission regions, especially in high-mass star-forming regions. If available, MHOs should have a near-infrared spectrum consistent with thermal (shock) excitation, rather than non-thermal (fluorescent) excitation (e.g. Gredel 1994; Lorenzetti et al. 2002; Caratti o Garatti et al. 2006; Gianninni et al. 2008). Kinematic studies - either proper motion studies (Hodapp 1999; Davis et al. 2009) or high spectral-resolution line studies (Carr 1993; Schwartz \& Greene 2003; Davis et al. 2004; Li et al. 2008) - are also useful for distinguishing MHOs from what are essentially stationary emission-line features in photon-dominated regions (PDRs). The association of an MHO with a bipolar molecular outflow, traced 


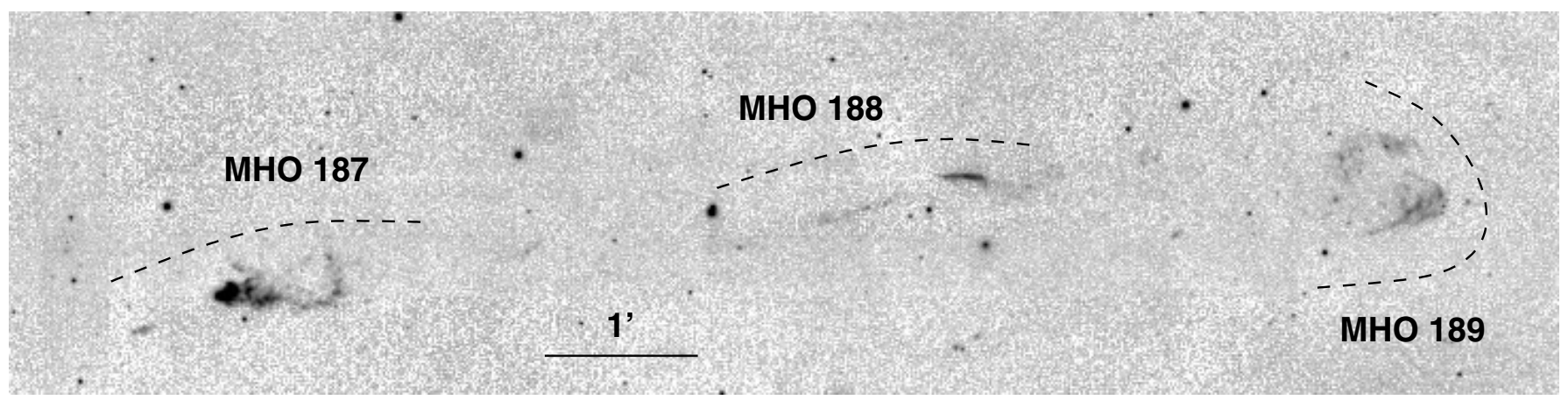

Fig. 1. $\mathrm{H}_{2}$ (+ continuum) image of the MHO 187-189 outflow in Orion A. Data from Davis et al. (2009).

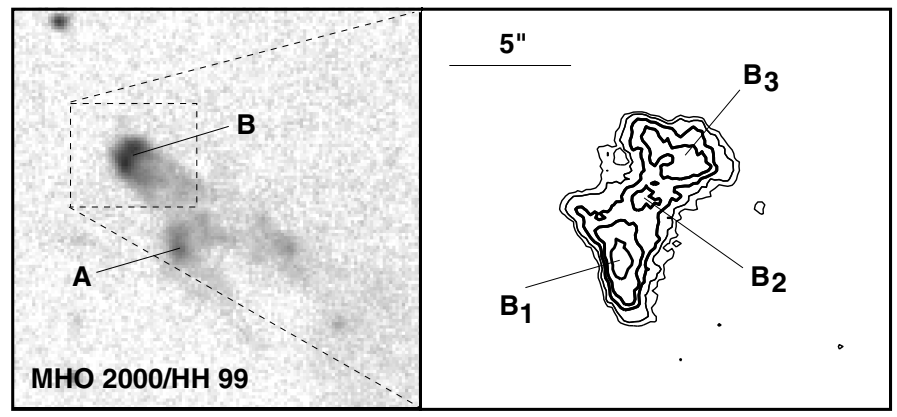

Fig. 2. A simple example of how knots and sub-knots within a single MHO should be labelled, using letters and, for the sub-knots, numbers.

in (sub)millimeter molecular lines such as CO, likewise confirms the dynamical nature and shock-excitation of the object, and its association with a protostar (e.g. Yu et al. 1999, 2000; Shepherd et al. 2003; Beuther et al. 2003; Reipurth et al. 2004).

The MHOs listed in this catalogue have all been identified in the near-infrared $(1-2.5 \mu \mathrm{m})$ lines of molecular hydrogen. Objects detected only in other near-IR lines (e.g. [FeII]) are not included. We also exclude objects observed only in the UV or mid-infrared (e.g. with the Spitzer Space Telescope). If an object is subsequently detected in molecular hydrogen line emission in the near-IR, it will be included in the MHO catalogue.

Examples of MHOs are shown in Figs. 1-3. In most cases we have labelled "groups of knots" rather than individual features or whole outflows. Assigning an MHO number to every resolved feature would of course lead to a vast catalogue that was impossible to maintain. On the other hand, associating widelyspaced knots with a single outflow is often difficult, given the variability of these line emission features and the large sizes of some outflows. MHO 187-189 (shown in Fig. 1) is a good example, where three complex groups of features that may well form part of the same outflow are none-the-less catalogued separately, although individual knots within each region are not. As with $\mathrm{HH}$ objects, if necessary, individual knots should be identified with letters; sub-knots should then be labelled with letters and numbers. HH 99 (MHO 2000) is shown as an example in Fig. 2 (see also the labelling of the knots and sub-features in the detailed, proper-motion study of HH 47/46 in Eislöffel \& Mundt 1994).

In some regions multiple knots and bow shocks radiate in many directions from a tight cluster of young stars. Since the relationship between these objects is often unclear - each bow shock may for example be driven by a different outflow that is powered by a different protostar in the central region - we also label these features separately. An example of such a region, the spectacular AFGL 961 massive star forming cluster in the Rosette nebula (described in detail by Aspin 1998; Li et al. 2008), is shown in Fig. 3.

Finally, for completeness we have also given a catalogue number to many well-known $\mathrm{HH}$ objects (e.g. HH 1/2 = MHO 120/125, HH 212 = MHO 499), though only if these are detected in the near-IR lines of molecular hydrogen. Whenever possible, we group features together in a manner consistent with the $\mathrm{HH}$ object catalogue.

\section{The MHO Catalogue}

\subsection{Grouping MHOs by region}

There are already almost 1000 objects in the MHO catalogue. In an attempt to bring some semblance of order to the list, we have grouped objects by "region".

Strictly speaking, there are no official names for, or boundaries to, the star-forming giant molecular clouds in our Galaxy. We have therefore attempted to define large regions based on the well-defined boundaries of the 88 constellations (as outlined by the $\mathrm{IAU}^{2}$ ). MHOs are almost exclusively confined to molecular clouds in and around the Gould belt and the Galactic plane (the vast majority of molecular outflows are driven by embedded protostars (Davis et al. 2008, 2009); relatively few T Tauri stars drive jets that have been detected in molecular hydrogen line emission, and of course $\mathrm{H}_{2}$ emission, by its very nature, requires the presence of dense molecular gas). We have therefore, in some areas, modified these boundaries slightly to include large groups of clouds. We use the large-scale CO $J=1-0$ survey of the Milky Way, obtained with $1.2 \mathrm{~m}$ telescopes in Cambridge, Massachusetts and Cerro Tololo, Chile (Dame et al. 2001) to identify these clouds. Even so, the boundaries will still pass through some smaller, less massive clouds and so the boundaries should only be considered accurate to within a few arcminutes.

The regions defined in this way are listed in the first column in Table 1. Note that, in the heavily-populated area of Orion, we have split the region up into two sub-regions, Orion A and Orion $\mathrm{B}$, as is the popular convention.

The boundaries of each region are also marked on lowresolution $\mathrm{CO} J=1-0$ maps in Fig. 4. M 1-M9 in these figures and in Table 1 refer to maps 1 to 9 . Note that not all 88 constellations are listed in Table 1, since those at high galactic latitudes do not contain star forming regions and/or known outflows with MHOs. Indeed, five regions; Camelopardalis, Centaurus,

\footnotetext{
2 http://www.iau.org/public_press/themes/ constellations/
} 


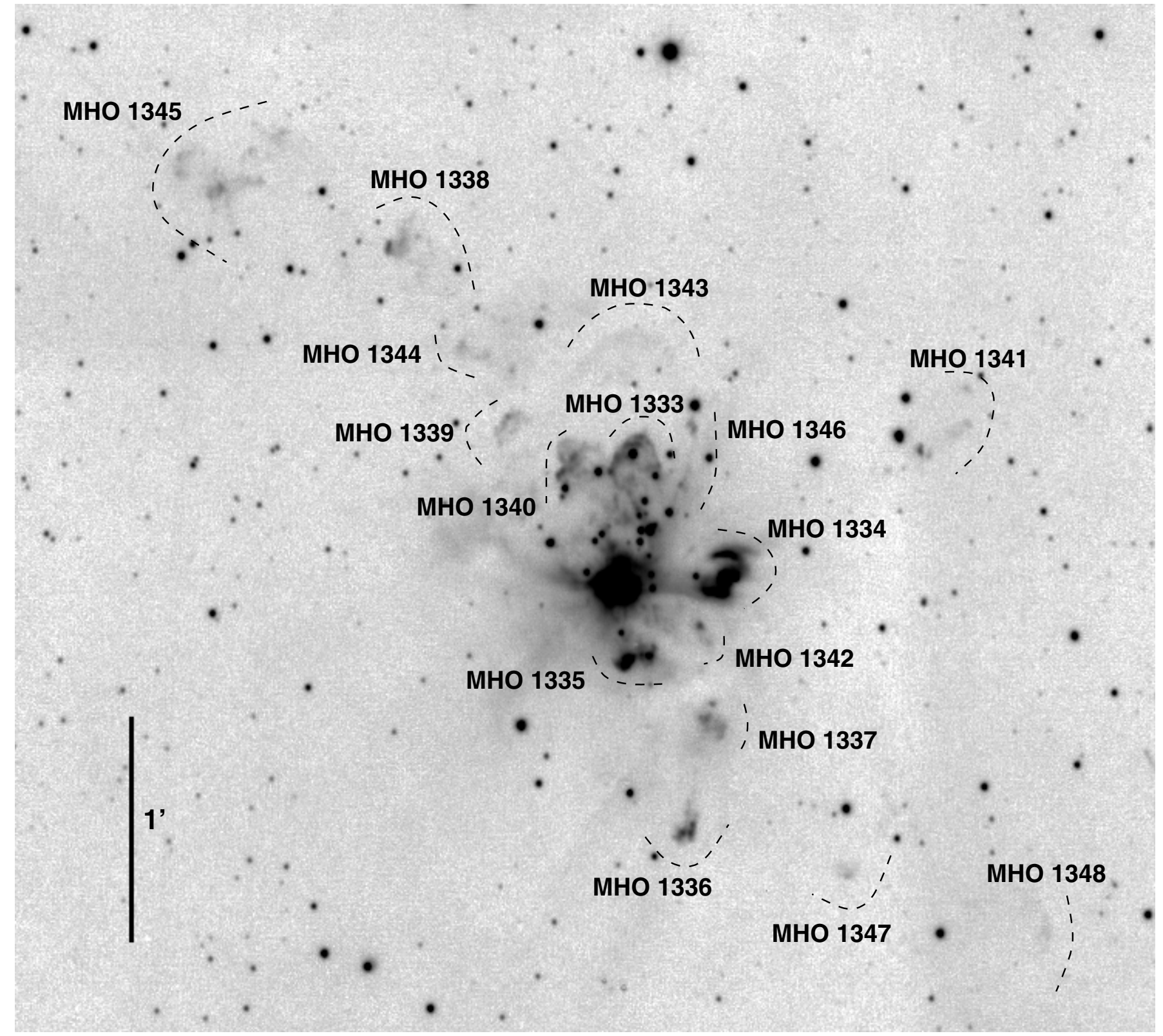

Fig. 3. $\mathrm{H}_{2}$ (+ continuum) image of AFGL 961 in the Rosette nebula star forming region in Monoceros. Catalogued MHOs are labelled; unpublished data obtained with WFCAM at UKIRT (see Davis et al. 2009 for details of this instrument, the WFCAM data archive and data processing techniques used to create this image).

Circinus/Lupus, Lyra and Andromeda, as yet contain no MHOs. We include these regions in the catalogue to facilitate the addition of future discoveries.

The MHO number range listed in the final column in Table 1 defines the range of MHO numbers currently being used in each region. To date, not all numbers have been assigned to an $\mathrm{MHO}$ (in any of the regions).

The latest version, at the time of writing, of the MHO catalogue is published here in Appendix A.

\subsection{The on-line database of MHOs}

The entire catalogue is also available on-line at http://wWw . jach.hawaii . edu/UKIRT/MHCat.
This MHO homepage includes the table of regions shown here in Table 1; in the on-line catalogue, links in the first column point to separate tables of MHOs for each region. These tables list the MHO number, right ascension and declination, citations to the discovery paper and subsequent near-IR imaging papers, together with identifications used in the literature, any associated $\mathrm{HH}$ objects, and a brief description of each object. A small image of the MHO is also presented with the object clearly marked; example images from the on-line catalogue are shown here in Fig. 5.

In the on-line catalogue simple ascii tables are also available. These list only MHO number, right ascension and declination, associated $\mathrm{HH}$ object, and region. These very basic tables may be downloaded and used to plot positions of MHOs on images or maps taken at other wavelengths, or to label $\mathrm{H}_{2}$ emission-line 
Table 1. Regions used to group MHOs.

\begin{tabular}{|c|c|c|c|c|}
\hline Region $^{a}$ & $\operatorname{Map}^{a}$ & Approx. RA Range ${ }^{b}$ & Approx. Dec Range ${ }^{b}$ & MHO Numbers ${ }^{c}$ \\
\hline Perseus & M2 & $03 \mathrm{~h} 00 \mathrm{~m} \rightarrow 04 \mathrm{~h} 00 \mathrm{~m}$ & $+25^{\circ} \rightarrow+35^{\circ}$ & $500-699$ \\
\hline Auriga & M2 & $03 \mathrm{~h} 30 \mathrm{~m} \rightarrow 06 \mathrm{~h} 30 \mathrm{~m}$ & $+30^{\circ} \rightarrow+56^{\circ}$ & 1000-1099 \\
\hline Taurus & M2 & $03 \mathrm{~h} 00 \mathrm{~m} \rightarrow 05 \mathrm{~h} 50 \mathrm{~m}$ & $+10^{\circ} \rightarrow+30^{\circ}$ & $700-799$ \\
\hline Camelopardalis & M 1 & $04 \mathrm{~h} 00 \mathrm{~m} \rightarrow 08 \mathrm{~h} 00 \mathrm{~m}$ & $+56^{\circ} \rightarrow+90^{\circ}$ & $1100-1199$ \\
\hline Orion A & M3 & $04 \mathrm{~h} 45 \mathrm{~m} \rightarrow 06 \mathrm{~h} 00 \mathrm{~m}$ & $-15^{\circ} \rightarrow-04^{\circ}$ & $1-299$ \\
\hline Orion B & M3 & $04 \mathrm{~h} 45 \mathrm{~m} \rightarrow 06 \mathrm{~h} 00 \mathrm{~m}$ & $-04^{\circ} \rightarrow+16^{\circ}$ & $300-499$ \\
\hline Gemini & M3 & $05 \mathrm{~h} 50 \mathrm{~m} \rightarrow 08 \mathrm{~h} 00 \mathrm{~m}$ & $+14^{\circ} \rightarrow+34^{\circ}$ & $1200-1299$ \\
\hline Monoceros & M3 & $06 \mathrm{~h} 00 \mathrm{~m} \rightarrow 08 \mathrm{~h} 30 \mathrm{~m}$ & $-13^{\circ} \rightarrow+14^{\circ}$ & $1300-1399$ \\
\hline Puppis & M4 & $06 \mathrm{~h} 30 \mathrm{~m} \rightarrow 09 \mathrm{~h} 00 \mathrm{~m}$ & $-38^{\circ} \rightarrow-13^{\circ}$ & $1400-1499$ \\
\hline Vela & M4 & $07 \mathrm{~h} 30 \mathrm{~m} \rightarrow 11 \mathrm{~h} 00 \mathrm{~m}$ & $-55^{\circ} \rightarrow-38^{\circ}$ & $1500-1599$ \\
\hline Carina & M5 & $08 \mathrm{~h} 00 \mathrm{~m} \rightarrow 12 \mathrm{~h} 00 \mathrm{~m}$ & $-75^{\circ} \rightarrow-55^{\circ}$ & $1600-1699$ \\
\hline Chameleon & M5 & $08 \mathrm{~h} 00 \mathrm{~m} \rightarrow 14 \mathrm{~h} 00 \mathrm{~m}$ & $-85^{\circ} \rightarrow-70^{\circ}$ & 3000-3099 \\
\hline Centaurus & M5 & $12 \mathrm{~h} 00 \mathrm{~m} \rightarrow 15 \mathrm{~h} 00 \mathrm{~m}$ & $-70^{\circ} \rightarrow-30^{\circ}$ & $1700-1799$ \\
\hline Circinus/Lupus & M6 & $15 \mathrm{~h} 00 \mathrm{~m} \rightarrow 16 \mathrm{~h} 00 \mathrm{~m}$ & $-70^{\circ} \rightarrow-30^{\circ}$ & 1800-1899 \\
\hline Scorpius & M6 & $16 \mathrm{~h} 00 \mathrm{~m} \rightarrow 18 \mathrm{~h} 00 \mathrm{~m}$ & $-60^{\circ} \rightarrow-30^{\circ}$ & 1900-1999 \\
\hline Corona Australis & M6 & $18 \mathrm{~h} 00 \mathrm{~m} \rightarrow 19 \mathrm{~h} 30 \mathrm{~m}$ & $-45^{\circ} \rightarrow-35^{\circ}$ & 2000-2099 \\
\hline Ophiuchus & M6 & $16 \mathrm{~h} 00 \mathrm{~m} \rightarrow 18 \mathrm{~h} 00 \mathrm{~m}$ & $-30^{\circ} \rightarrow+05^{\circ}$ & 2100-2199 \\
\hline Serpens & M7 & $17 \mathrm{~h} 30 \mathrm{~m} \rightarrow 18 \mathrm{~h} 40 \mathrm{~m}$ & $-15^{\circ} \rightarrow+05^{\circ}$ & $2200-2299$ \\
\hline Sagittarius & M7 & $18 \mathrm{~h} 00 \mathrm{~m} \rightarrow 20 \mathrm{~h} 30 \mathrm{~m}$ & $-35^{\circ} \rightarrow-12^{\circ}$ & $2300-2399$ \\
\hline Aquila & M7 & $18 \mathrm{~h} 40 \mathrm{~m} \rightarrow 20 \mathrm{~h} 30 \mathrm{~m}$ & $-12^{\circ} \rightarrow+15^{\circ}$ & $2400-2499$ \\
\hline Lyra & M 8 & $18 \mathrm{~h} 20 \mathrm{~m} \rightarrow 19 \mathrm{~h} 00 \mathrm{~m}$ & $+05^{\circ} \rightarrow+45^{\circ}$ & $2500-2599$ \\
\hline Vulpecula & M 8 & $19 \mathrm{~h} 00 \mathrm{~m} \rightarrow 21 \mathrm{~h} 30 \mathrm{~m}$ & $+15^{\circ} \rightarrow+30^{\circ}$ & $2600-2699$ \\
\hline Cygnus & M9 & $19 \mathrm{~h} 00 \mathrm{~m} \rightarrow 22 \mathrm{~h} 00 \mathrm{~m}$ & $+30^{\circ} \rightarrow+55^{\circ}$ & $800-999$ \\
\hline Cepheus & M9 & $19 \mathrm{~h} 00 \mathrm{~m} \rightarrow 23 \mathrm{~h} 30 \mathrm{~m}$ & $+55^{\circ} \rightarrow+90^{\circ}$ & $2700-2799$ \\
\hline Andromeda & M9 & $22 \mathrm{~h} 00 \mathrm{~m} \rightarrow 00 \mathrm{~h} 00 \mathrm{~m}$ & $+30^{\circ} \rightarrow+55^{\circ}$ & $2800-2899$ \\
\hline Cassiopeia & M 1 & $23 \mathrm{~h} 00 \mathrm{~m} \rightarrow 04 \mathrm{~h} 00 \mathrm{~m}$ & $+50^{\circ} \rightarrow+90^{\circ}$ & 2900-2999 \\
\hline
\end{tabular}

Notes. ${ }^{(a)}$ The name of each region, and the map used to define each region; ${ }^{(b)}$ approximate RA and Dec range associated with each region (a more precise range is drawn on each map in Figs. 4; ${ }^{(c)}$ the range of MHO numbers used for objects within each region (note that objects have not yet been assigned to all numbers in each range).

features in new near-IR images of star forming regions already covered by the catalogue.

\subsection{Searching through the catalogue}

An easy way to navigate through the catalogue and, in particular, to search for objects by right ascension and declination, was thought to be desirable. A Perl script has therefore been developed which allows the user to enter coordinates and a search radius; the script returns an HTML table containing MHOs found within the search area. As with the full region tables, coordinates, references to published observations, a small image and a brief description of each object is returned. This tool is particularly useful for finding MHOs in a star forming cloud or cluster being studied at different wavelengths, or for establishing whether an object is a new discovery, or has in fact already been observed.

\subsection{Checking the catalogue}

Duplicating existing entries and errors associated with the coordinates assigned to each $\mathrm{MHO}$ were our two main concerns when compiling the catalogue. To combat both problems, the ascii text files created for each region were imported into the STARLINK GAIA graphical display tool (Draper et al. 2008) and plotted over wide field $R$-band Digitised Sky Survey (DSS) images or, if available, astrometrically-calibrated infrared images. The infrared images were all obtained from the UKIRT WFCAM archive $^{3}$.

\subsection{The future}

Our aim is to keep the MHO catalogue as up-to-date as possible. Also, obviously we want to avoid duplication of catalogue numbers (people using the same numbers for different objects). Therefore, we ask that those with new observations please check the catalogue for previous observations, and contact the catalogue organisers (currently Chris Davis: c.davis@jach.hawaii.edu) before papers are written, and certainly before figures and tables of MHOs are finalised, so that new numbers can be assigned.

$\overline{{ }^{3} \text { http://surveys.roe.ac.uk/wsa/index.html }}$ 

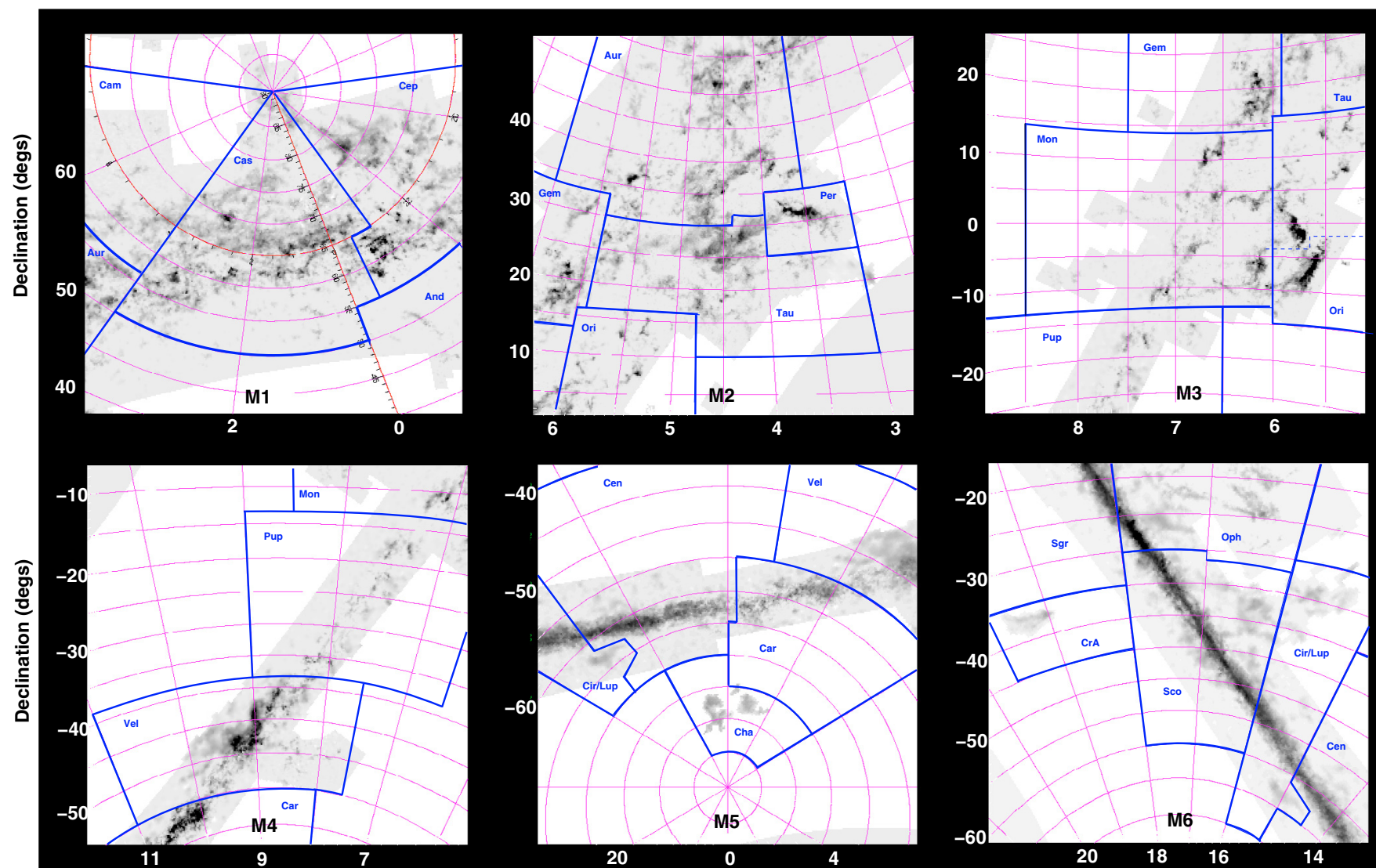

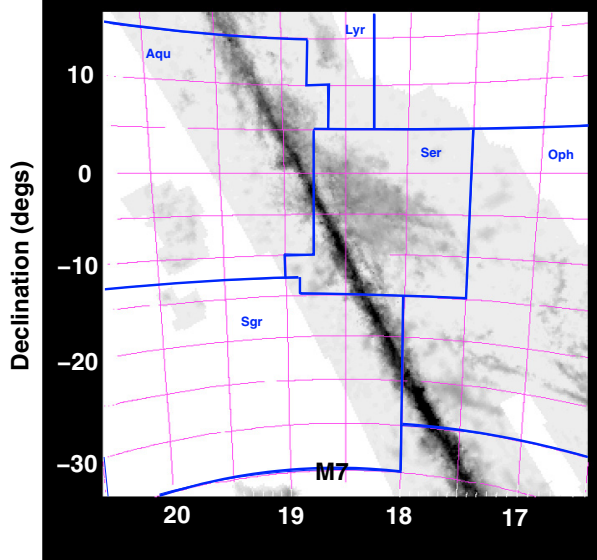

Right Ascension (hrs)

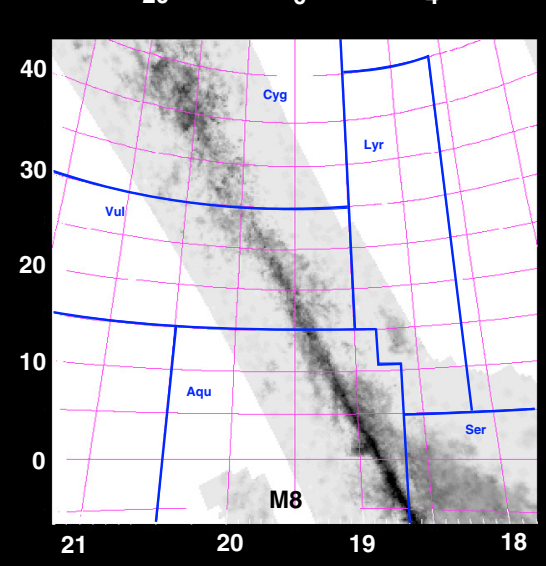

Right Ascension (hrs)

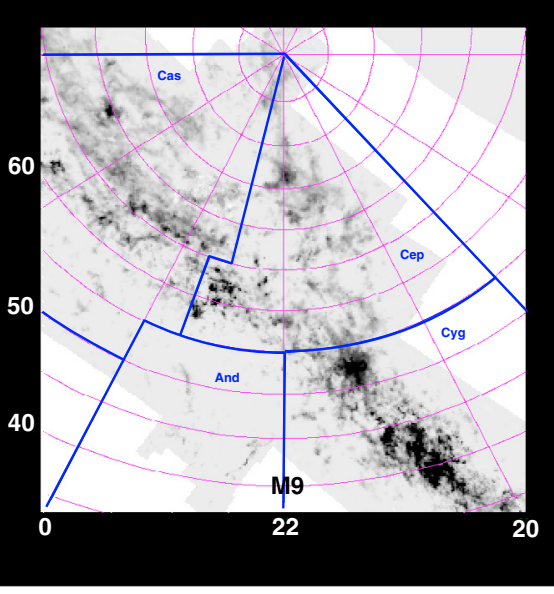

Right Ascension (hrs)

Fig. 4. Large-scale maps in CO $J=1-0$ emission with the boundaries of the regions used to group MHOs marked with thick lines.

\section{Summary}

A catalogue of molecular hydrogen emission-line objects (MHOs) has been compiled from the literature. The catalogue includes objects imaged in molecular hydrogen line emission (almost entirely in the $1-0 \mathrm{~S}(1)$ line at $2.122 \mu \mathrm{m}$ ). It does not include objects observed only at UV or mid-IR wavelengths.

The catalogue lists only shock-excited features associated with outflows from young stars. Objects in both low and highmass star forming regions are included. Similar objects associated with proto-planetary nebulae or extra-galactic sources are not included.
The catalogue currently contains almost 1000 objects. Some are well-known Herbig-Haro objects which we have included for completeness. The catalogue is available on-line at http:// Www . jach.hawaii.edu/UKIRT/MHCat/. With the help of the star formation community, we aim to maintain this catalogue for many years to come, adding new objects as they are discovered. We also hope that in the future, the MHO acronym will be used universally when labelling these enigmatic objects.

Acknowledgements. We thank the "Clearing House" of the Commission 5 Working Group on Designations, particularly the chair, Marion Schmitz, for their guidance, and the star formation community, especially Bo Reipurth, for their valuable input. This project would not have been possible without support from the Joint Astronomy Centre. 

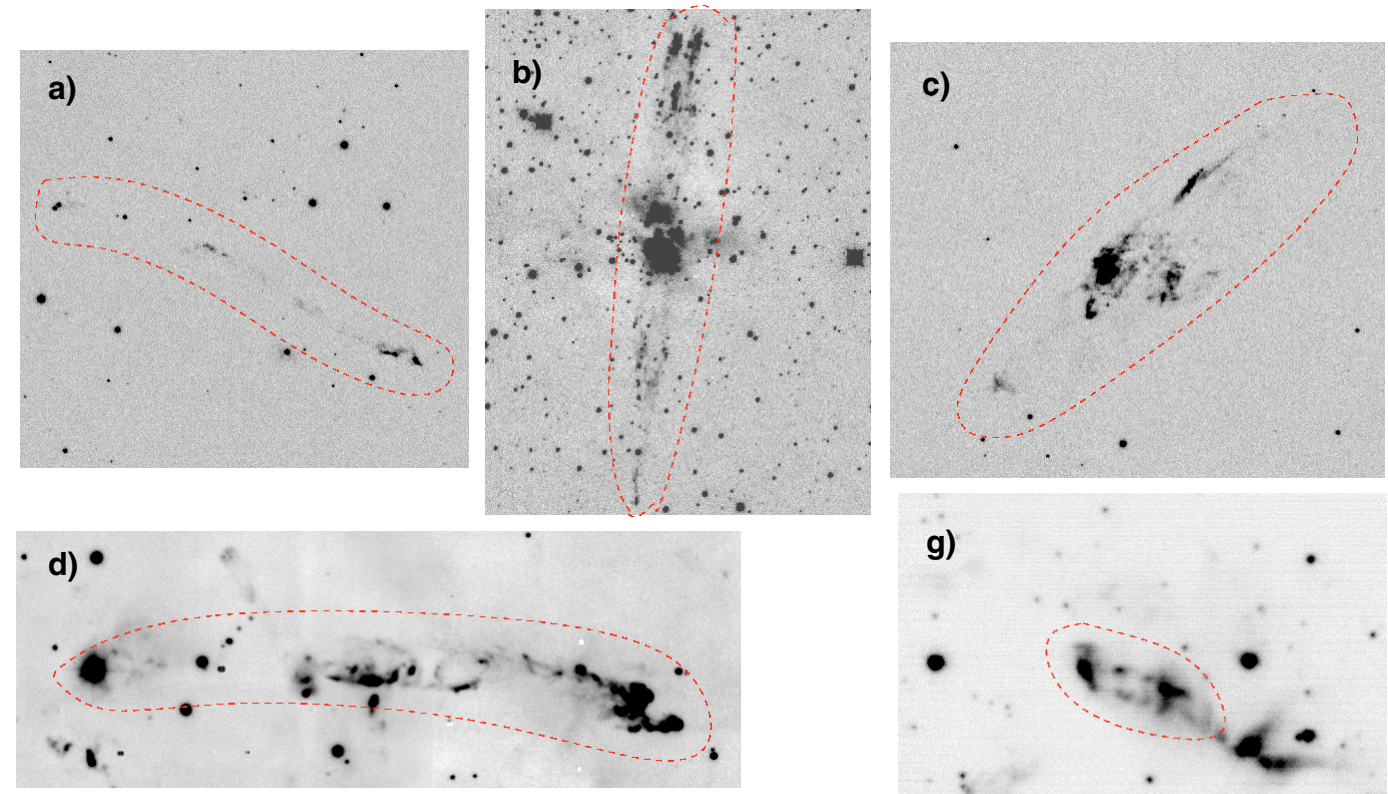

e)
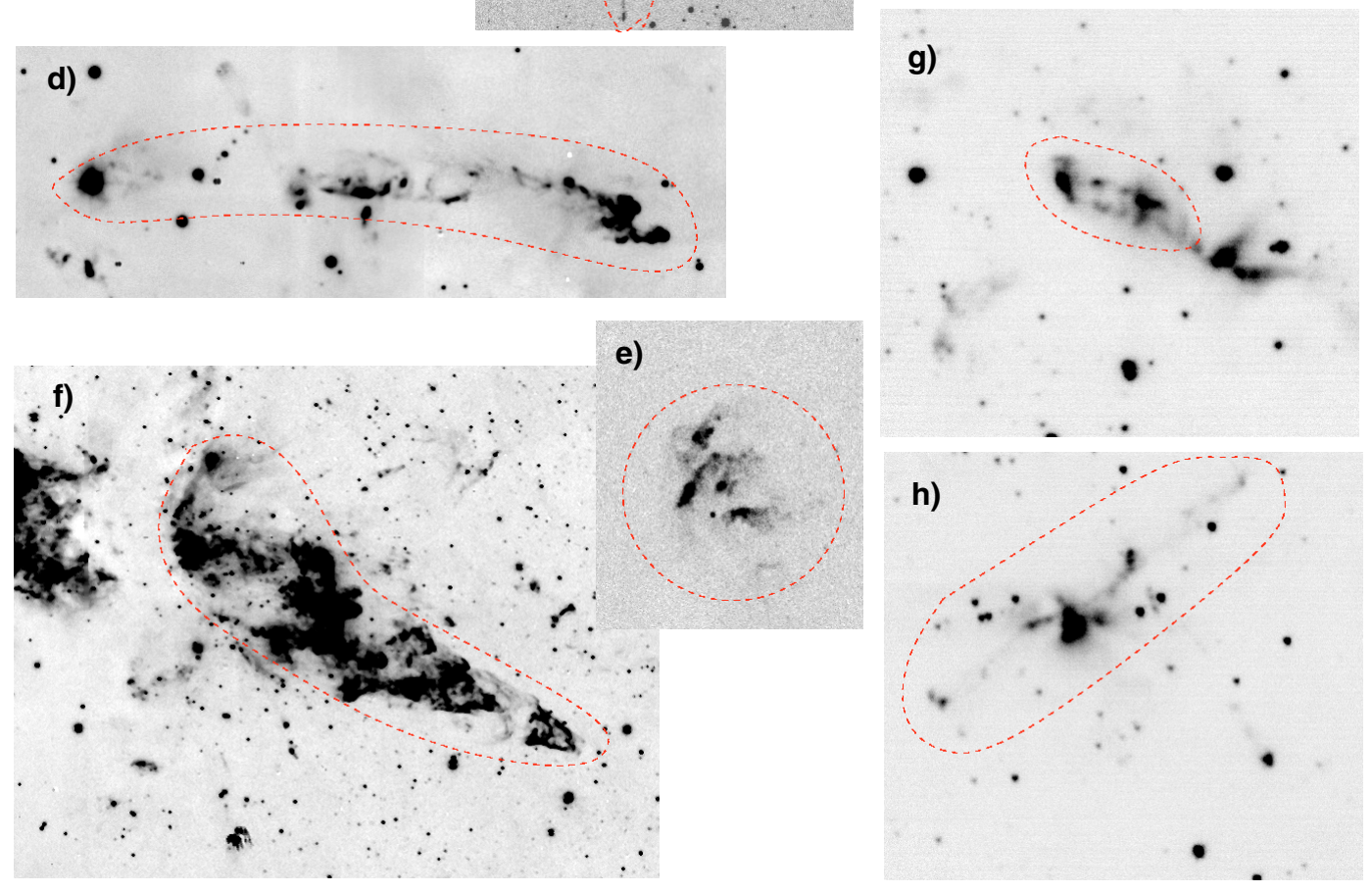

Fig. 5. Examples of the small images available at the MHO web site; in each case the MHO is marked with a red dashed ellipse or circle: a) MHO 1300, a curving, collimated jet $\sim 5^{\prime} \mathrm{SW}$ of the main Mon R2 star forming region in Monoceros (from Hodapp 2007); b) MHO 1510, a bipolar outflow associated with the bright, nebulous source IRS 20 in Vela (Giannini et al. 2007); c) MHO 558 (HH 773), a bright, knotty feature in a bipolar molecular outflow in the B1 ridge in Perseus (Walawender et al. 2009); d) MHO 18, a spectacular, knotty outflow in the OMC $2 / 3$ region in Orion A (Yu et al. 1997); e) MHO 3000, arcs of emission associated with HH 54 in Chameleon (Zealey et al. 1993); f) MHO 899, the luminous south-western molecular flow lobe associated with DR 21 in Cygnus (Davis \& Smith 1996); g) and h) the collimated outflows MHO 2604 and MHO 2201, associated with the high-mass star forming regions IRAS 19410+2336 in Vulpecula and IRAS 18151-1208 in Serpens, respectively (Varricatt et al. 2010).

\section{References}

Anandarao, B. G., Chakraborty, A., Ojha, D. K., et al. 2004, A\&A, 421, 1045

Aspin, C. 1998, A\&A, 335, 1040

Aspin, C., Sandell, G., \& Russell, A. P. G. 1994, A\&AS, 106, 165

Ayala, S., Noriega-Crespo, A., Garnavich, P. M., et al. 2000, AJ, 120, 909

Bachiller, R., Terebey, S., Jarrett, T., et al. 1994, ApJ, 437, 296

Bally, J., Devine, D., Hereld, M., et al. 1993a, ApJ, 418, L75

Bally, J., Lada, E. A., \& Lane, A. P. 1993b, ApJ, 418, 322

Bally, J., Devine, D., Fesen, R. A., et al. 1995, ApJ, 454, 345

Beck, T. L., McGregor, P. J., Takami, M., et al. 2008, ApJ, 676, 427

Beuther, H., Schilke, P., \& Stanke, T. 2003, A\&A, 408, 601

Birkmann, S. M., Krause, O., Hennemann, M., et al. 2007, A\&A, 474, 883

Bontemps, S., Ward-Thompson, D., \& André, P. 1996, A\&A, 314, 477

Brooks, K. J., Garay, G., Mardones, D., et al. 2003, ApJ, 594, L131

Bourke, T. L. 2001, ApJ, 554, L91

Caratti o Garatti, A., Giannini, T., Lorenzetti, D., et al. 2004, A\&A, 422, 14

Caratti o Garatti, A., Giannini, T., Nisini, B., et al. 2006, A\&A, 449, 1077

Caratti o Garatti, A., Froebrich, D., Eislöffel, J., et al. 2008, A\&A, 485, 137

Caratti o Garatti, A., Eislöffel, J., Froebrich, D., et al. 2009, A\&A, 502, 579

Carr, J. S. 1993, ApJ, 406, 553

Cesaroni, R., Neri, R., Olmi, L., et al. 2005, A\&A, 434, 1039
Chakraborty, A., Ojha, D. K., Anandarao, B. G., et al. 2000, A\&A, 364, 683 Chen, X.-P., \& Yao, Y.-Q. 2004, ChJA\&A, 4, 284

Chen, Y., Yao, Y., Yang, J., et al. 1999, AJ, 117, 446

Chen, Y., Zheng, X.-W., Yao, Y., Yang, J., \& Sato, S. 2003, A\&A, 401, 185

Chen, Y., Yao, Y., Yang, J., Zeng, Q., \& Sato, S. 2009, ApJ, 693, 430

Choi, M., Hodapp, K. W., Hayashi, M., et al. 2006, ApJ, 646, 1050

Chrysostomou, A., Hobson, J., Davs, C. J., Smith, M. D., \& Berndsen, A. 2000, MNRAS, 314, 229

Codella, C., Cabrit, S., Gueth, F., et al. 2007, A\&A, 462, L53

Connelley, M. S., Reipurth, B., \& Tokunaga, A. T. 2007, AJ, 133, 1528

Coppin, K. E. K., Davis, C. J., \& Micono, M. 1998, MNRAS, 301, L10

Cruz-González, I., Salas, L., \& Hiriart, D. 2007, RMA\&A, 43, 337

Cunningham, N. J., Moeckel, N., \& Bally, J. 2009, ApJ, 692, 943

Dame, T. M., Hartmann, D., \& Thaddeus, P. 2001, ApJ, 555, 12

Davis, C. J., \& Eislöffel, J. 1995, A\&A, 300, 851

Davis, C. J., \& Smith, M. D. 1995, ApJ, 443, L41

Davis, C. J., \& Smith, M. D. 1996, A\&A, 310, 961

Davis, C. J., Dent, W. R. F., Mathews, H. E., Aspin, C., \& Lightfoot, J. F. 1994a, MNRAS, 266, 933

Davis, C. J., Eislöffel, J., \& Ray, T. P. 1994b, ApJ, 426, L93

Davis, C.J., Mundt, R., \& Eislöffel, J. 1994c, ApJ, 437, L55

Davis, C. J., Mundt, R., Eislöffel, J., et al. 1995, AJ, 110, 766

Davis, C. J., Eislöffel, J., \& Smith, M. D. 1996, ApJ, 463, 246 
Davis, C. J., Ray, T. P., Eislöffel, J., et al. 1997, A\&A, 324, 263

Davis, C. J., Moriarty-Schieven, G., Eislöffel, J., Hoare, M. G., \& Ray, T. P. 1998, AJ, 115, 1118

Davis, C. J., Smith, M. D., Eislöffel, J., et al. 1999, MNRAS, 308, 539

Davis, C. J., Dent, W. R. F., Mathews, H. E., Coulson, I. M., \& McCaughrean, M. J. 2000a, MNRAS, 318, 952

Davis, C. J., Smith, M. D., \& Eislöffel, J. 2000b, MNRAS, 318, 747

Davis, C. J., Stern, L., Ray, T. P., et al. 2002, A\&A, 382, 1021

Davis, C. J., Varricatt, W. P., Todd, S. P., et al. 2004, A\&A, 425, 981

Davis, C. J., Kumar, M. S. N., Sandell, G., et al. 2007, MNRAS, 374, 29

Davis, C. J., Scholz, P., Lucas, P., Smith, M. D., \& Adamson, A. 2008, MNRAS, 387,954

Davis, C. J., Froebrich, D., Stanke, T., et al. 2009, A\&A, 496, 153

Davis, C. J., et al. 2010, MNRAS, submitted

De Luca, M., Elia, D., Giannini, T., et al. 2007, A\&A 474, 863

Dent, W. R. F., Matthews, H. E., \& Walther, D. M. 1995, MNRAS, 277, 193

Djupvik, A. A., André, Ph., Bontemps, S., et al. 2006, A\&A, 458, 789

Draper, P. W., Berry, D. S., Jenness, T., Economou, F., \& Currie, M. J. 2008, ASPC, 394, 339

Eislöffel, J. 2000, A\&A, 354, 236

Eislöffel, J., \& Mundt, R. 1994, A\&A, 284, 530

Eislöffel, J., Smith, M. D., Davis, C.J., et al. 1996, AJ, 112, 2086

Eislöffel, J., Davis, C. J., Ray, T. P, et al. 1994, ApJ, 422, L91

Eislöffel, J., Froebrich, D., Stanke, T., et al. 2003, ApJ, 595, 259

Everett, M. E. 1997, ApJ, 478, 246

Fang, M., \& Yao, Y.-Q. 2004, ChA\&A, 28, 308

Fontani, F., Cesaroni, R., Testi, L., et al. 2004, A\&A 424, 179

Froebrich, D., \& Scholz, A. 2003, A\&A, 407, 207

Fuller, G. A., Lada, E. A., Masson, C. R., et al. 1995, ApJ, 453, 754

Gålfalk, M., \& Olofsson, G. 2007a, A\&A, 466, 579

Gålfalk, M., \& Olofsson, G. 2007b, A\&A, 475, 281

Gålfalk, M., \& Olofsson, G. 2008, A\&A, 489, 1409

Garden, R. P., Russell, A. P. G., \& Burton, M. G. 1990, ApJ, 354, 232

Garnavich, P. M., Noriega-Crespo, A., Raga, A. C., et al. 1997, ApJ, 490, 752

Giannini, T., Massi, F., Podio, L., et al. 2005, A\&A, 433, 941

Giannini, T., McCoey, C., Nisini, B., et al. 2006, A\&A, 459, 821

Giannini, T., Lorenzetti, D., De Luca, M., et al. 2007, ApJ, 671, 470

Giannini, T., Calzoletti, L., Nisini, B., et al. 2008, A\&A, 481, 123

Ginsburg, A. G., Bally, J., Yan, C.-H., et al. 2009, ApJ, 707, 310

Goetz, J. A., Pipher, J. L., Forrest, W. J., et al. 1998, AJ, 504, 359

Gómez, M., Whitney, B. A., \& Kenyon, S. J. 1997, AJ, 114, 1138

Gómez, M., Stark, D. P., Whitney, B. A., et al. 2003, AJ, 126, 863

Gómez, M., Persi, P., Marenzi, A. R., Roth, M., \& Tapia, M. 2004, A\&A, 423, 629

Gredel, R. 1994, A\&A, 292, 580

Gredel, R. 2006, A\&A, 457, 157

Gredel, R., \& Reipurth, B. 1993, ApJ, 407, L29

Gredel, R., \& Reipurth, B. 1994, A\&A, 289, L19

Grosso, N., Alves, J., Neuhauser, R., et al. 2001, A\&A, 380, L1

Hartigan, P., Carpenter, J. M., Dougados, C., et al. 1996, AJ, 111, 1278

Hartigan, P., Morse, J., \& Bally, J. 2000, AJ, 120, 1436

Hayashi, M., \& Pyo, T. S. 2009, ApJ, 694, 582

Herbst, T. M., Beckwith, S. V. W., \& Robberto, M. 1997, ApJ, 486, L59

Herbst, T. M., Hartung, M., Kasper, M. E., Leinert, C., \& Ratzka, T. 2007, AJ, 134,359

Hiriart, D., Salas, L., \& Cruz-González, I. 2004, AJ, 128, 2917

Hodapp, K. W. 1998, ApJ, 500, L183

Hodapp, K. W. 1999, AJ, 118, 1338

Hodapp, K. W. 2007, AJ, 134, 2020

Hodapp, K. W., \& Davis, C. J. 2002, ApJ, 575, 291

Hodapp, K. W., \& Ladd, E. F. 1995, ApJ, 453, 715

Hodapp, K. W., Bally, J., Eislöffel, J., et al. 2005, AJ, 129, 1580

Indebetouw, R., Watson, C., Johnson, K. E., Whitney, B., \& Churchwell, E., 2003, ApJ, 596, L83

Jiang, Z., Yao, Y., Yang, J., et al. 2003, ApJ, 596, 1064

Jiang, Z., Yang, J., Yao, Y., Ishii, M., \& Mao, R. 2004, ChA\&A, 28, 299

Khanzadyan, T., Smith, M. D., Davis, C. J., et al. 2003, MNRAS, 338, 57

Khanzadyan, T., Gredel, R., Smith, M. D., et al. 2004a, A\&A, 426, 171

Khanzadyan, T., Smith, M. D., Davis, C. J., et al. 2004b, A\&A, 418, 163

Kumar, M. S. N., Bachiller, R., \& Davis, C. J. 2002, ApJ, 576, 313

Ladd, E. F., \& Hodapp, K. W. 1997, ApJ, 475, 749

Li, J. Z., Smith, M. D., Gredel, R., Davis, C. J., \& Rector, T. A. 2008, ApJ, 679, L101

Lorenzetti, D., Giannini, T., Vitali, F., Massi, F., \& Nisini, B. 2002, ApJ, 564, 839

Lucas, P. W., Blundell, K. M., \& Roche, P. F. 2000, MNRAS, 318, 526

Massi, F., Codella, C., \& Brand, J. 2004, A\&A, 419, 241

McCaughrean, M. J., Rayner, J. T., \& Zinnecker, H. 1994, ApJ, 436, L189
Megeath, S. T., \& Tieftrunk, A. R. 1999, ApJ, 526, L113

Micono, M., Davis, C. J., Ray, T., Eislöffel, J., \& Shetrone, M. D. 1998, ApJ, 494, L227

Miralles, M. P., Salas, L., Cruz-González, I., et al. 1997, ApJ, 488, 749

Moreira, M. C., \& Yun, J. L. 1995, ApJ, 454, 850

Nadeau, D., Murphy, D. C., Doyon, R., et al. 1994, PASP, 106, 909

Nisini, B., Massi, F., Vitali, F., et al. 2001, A\&A, 376, 553

Noriega-Crespo, A., \& Garnavich, P. M. 1994, AJ, 108, 1432

Noriega-Crespo, A., Garnavich, P. M., Raga, A., Cantó, J., \& Böhm, K.-H. 1996, ApJ, 462, 804

Noriega-Crespo, A., Garnavich, P. M., Curiel, S., Raga, A., \& Ayala, S. 1997, ApJ, 486, L55

Noriega-Crespo, A., Cotera, A., Young, E., et al. 2002, ApJ, 580, 959

Nürnberger, D. E. A., Chini, R., Eisenhauer, F., et al. 2007, A\&A, 465, 931

O'Connell, B., Smith, M. D., Davis, C. J., et al. 2004, A\&A, 419, 975

O'Connell, B., Smith, M. D., Froebrich, D., Davis, C.J., \& Eislöffel, J. 2005, A\&A, 431, 223

Palacios, J., \& Eiroa, C. 1999, A\&A, 346, 233

Persi, P., Roth, M., Tapia, M., et al. 1996, A\&A, 307, 591

Persi, P., Tapia, M., Roth, M., et al. 2003, A\&A, 397, 227

Persi, P., Tapia, M., Roth, M., et al. 2009, A\&A, 493, 571

Phelps, R. L., \& Ybarra, J. E. 2005, ApJ, 627, 845

Piché, F., Howard, E. M., \& Pipher, J. L. 1995, MNRAS, 275, 711

Porras, A., Cruz-González, I., \& Salas, L. 2000, A\&A, 361, 660

Reach, W. T., Faied, D., Rho, J., et al. 2009, ApJ, 690, 683

Reipurth, B., Hartigan, P., Heathcote, S., Morse, J. A., \& Bally, J. 1997, AJ, 114, 757

Reipurth, B., Yu, K. C., Rodríguez, L. F., et al. 1999, A\&A, 352, L83

Reipurth, B., Heathcote, S., Yu, K. C., Bally, J., \& Rodríguez, L. F. 2000a, ApJ, 534, 317

Reipurth, B., Yu, K. C., Heathcote, S., Bally, J., \& Rodríguez, L. F. 2000b, AJ, 120,1449

Reipurth, B., Yu, K. C., Moriarty-Schieven, G., et al. 2004, AJ, 127, 1069

Salas, L., Cruz-González, I., \& Porras, A. 1998, ApJ, 500, 853

Salas, L., Cruz-González, I., \& Rosado, M. 2003, Rev. Mex. Astron. Astrofis., 39,77

Schultz, A. S. B., Rank, D., Temi, P., et al. 1995, Ap\&SS, 233, 71

Schwartz, R. D., \& Greene, T. P. 1999, AJ, 117, 456

Schwartz, R. D., \& Greene, T. P. 2003, AJ, 126, 339

Schwartz, R. D., Burton, M. G., \& Herrmann, J. 1997, AJ, 114, 272

Shepherd, D. S., Yu, K. C., Bally, J., et al. 2000, ApJ, 535, 833

Shepherd, D. S., Testi, L., \& Stark, D. P. 2003, AJ, 584, 882

Smith, H. A., \& Fischer, J. 1992, ApJ, 398, L99

Smith, M. D., Froebrich, D., \& Eislöffel, J. 2003a, ApJ, 592, 245

Smith, M. D., Khanzadyan, T., \& Davis, C.J. 2003b, MNRAS, 339, 524

Smith, M. D., O'Connell, B., \& Davis, C. J. 2007, A\&A, 466, 565

Stanke, T., McCaughrean, M. J., \& Zinnecker, H. 1998, A\&A, 332, 307

Stanke, T., McCaughrean, M. J., \& Zinnecker, H. 2002, A\&A, 392, 239

Stapelfeldt, K. R., Beichman, C. A., Hester, J. J., Scoville, N. Z., \& Gautier, T. N. 1991, ApJ, 371, 226

Tachihara, K., Rengel, M., Nakajima, Y., et al. 2007, ApJ, 659, 1382

Tamura, M., \& Yamashita, T. 1992, ApJ, 391, 710

Todd, S. P., \& Ramsay Howat, S. K. 2006, MNRAS, 367, 238

Tokunaga, A. T., Dahm, S., Gässler, W., et al. 2004, ApJ, 601, L91

Varricatt, W. P., Davis, C. J., \& Adamson, A. J. 2005, MNRAS, 359, 2

Varricatt, W. P., Davis, C. J., Ramsay, S., et al. 2010, MNRAS, in press

van Langevelde, H. J., van Dishoeck, E. F., van der Werf, P. P., et al. 1994, A\&A, 287, L25

Walawender, J., Bally, J., Kirk, H., et al. 2005, AJ, 130, 1795

Walawender, J., Bally, J., Kirk, H., et al. 2006, AJ, 132, 467

Walawender, J., Reipurth, B., \& Bally, J. 2009, AJ, 137, 3254

Wang, H., Yang, J., Wang, M., et al. 2002, A\&A, 389, 1015

Wang, H., Stecklum, B., \& Henning, Th. 2005, A\&A, 437, 169

Wilking, B. A., Schwartz, R. D., Mundy, L. G., et al. 1990, AJ, 99, 344

Wilking, B. A., McCaughrean, M. J., Burton, M. G., et al. 1997, AJ, 114, 2029

Yamashita, T., \& Tamura, M. 1992, ApJ, 387, L93

Yao, Y., Chen, Y., Yang, J., Takanori, H., \& Tetsuya, N. 2000, Acta Astron. Sin., 41,2

Ybarra, J. E., Barsony, M., Haisch, K. E., et al. 2006, ApJ, 647, L159

Yu, K. C., Bally, J., \& Devine, D. 1997, ApJ, 485, L45

Yu, K. C., Billawala, Y., \& Bally, J. 1999, AJ, 118, 2940

Yu, K. C., Smith, D. M., Bally, J., et al. 2000, AJ, 1974, 120

Yun, J. L., Clemens, D. P., Moreira, M. C., et al. 1997, ApJ, 479, L71

Yun, J. L., Santos, C. A., Clemens, D. P., et al. 2001, A\&A, 372, L33

Zealey, W. J., Williams, P. M., Sandell, G., Taylor, K. N. R., \& Ray, T. P. 1992, A\&A, 262, 570

Zealey, W. J., Suters, M. G., \& Randall, P. R. 1993, PASA, 10, 203

Zinnecker, H., McCaughrean, M. J., \& Rayner, J. T. 1998, Nature, 394, 862 


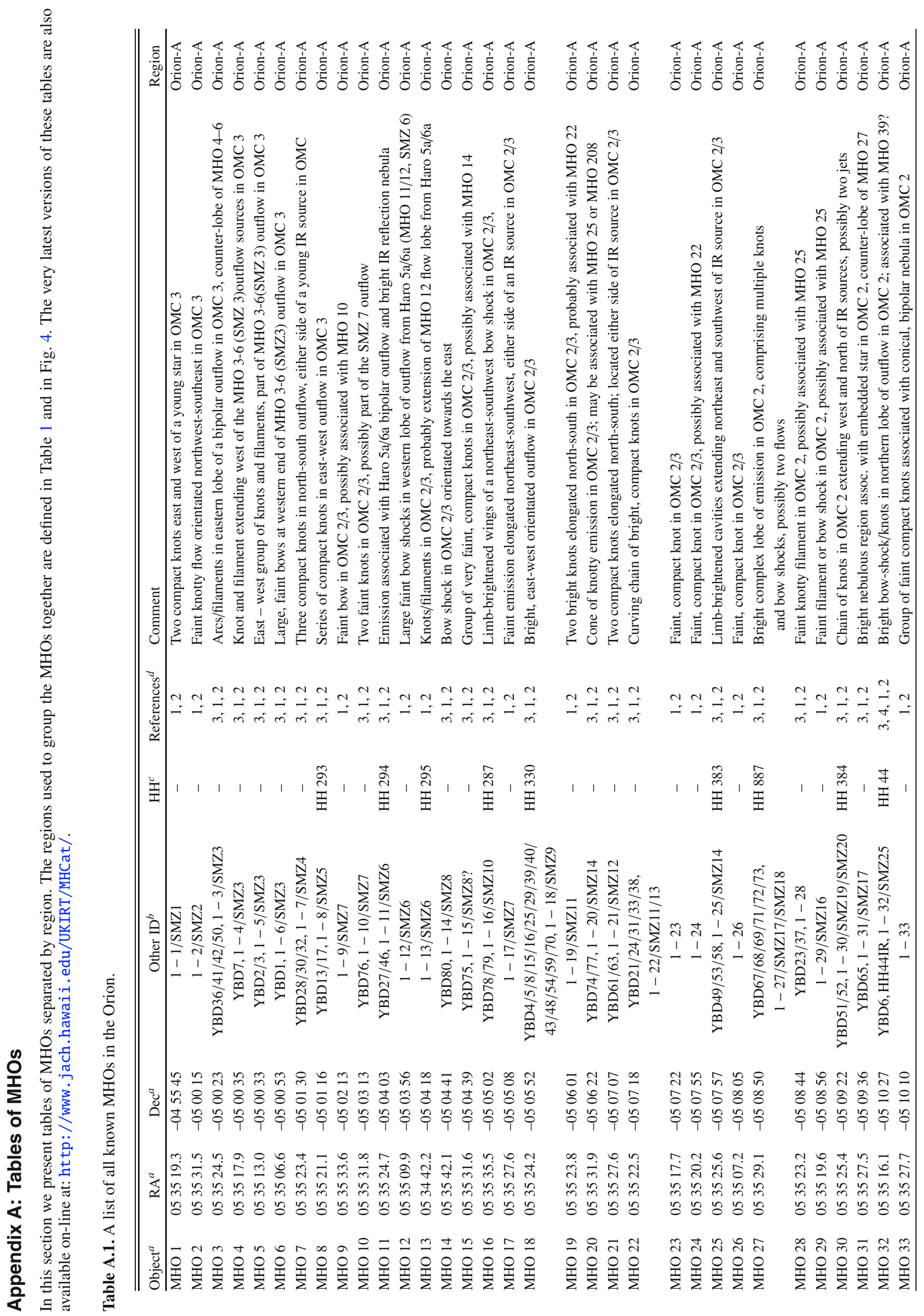




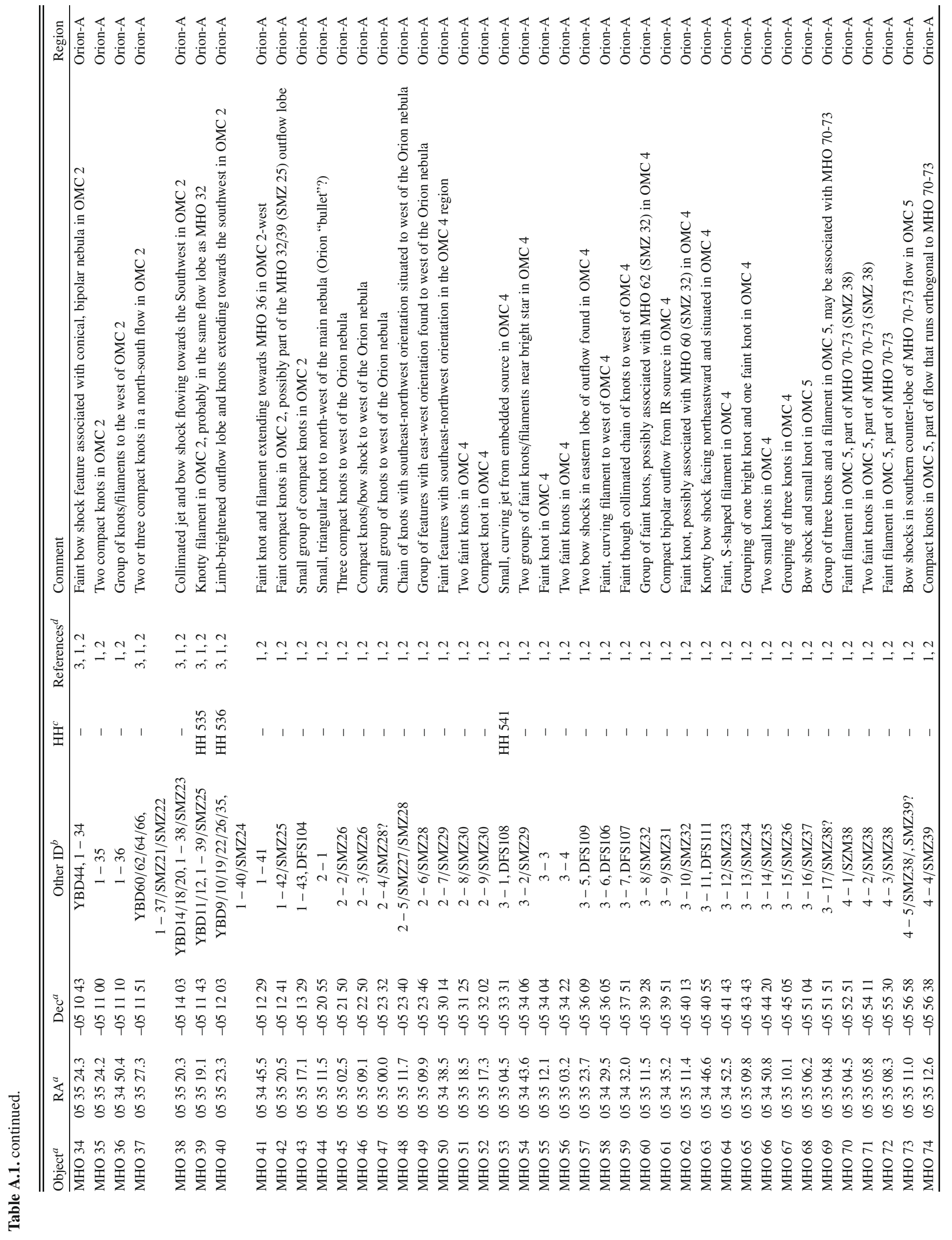




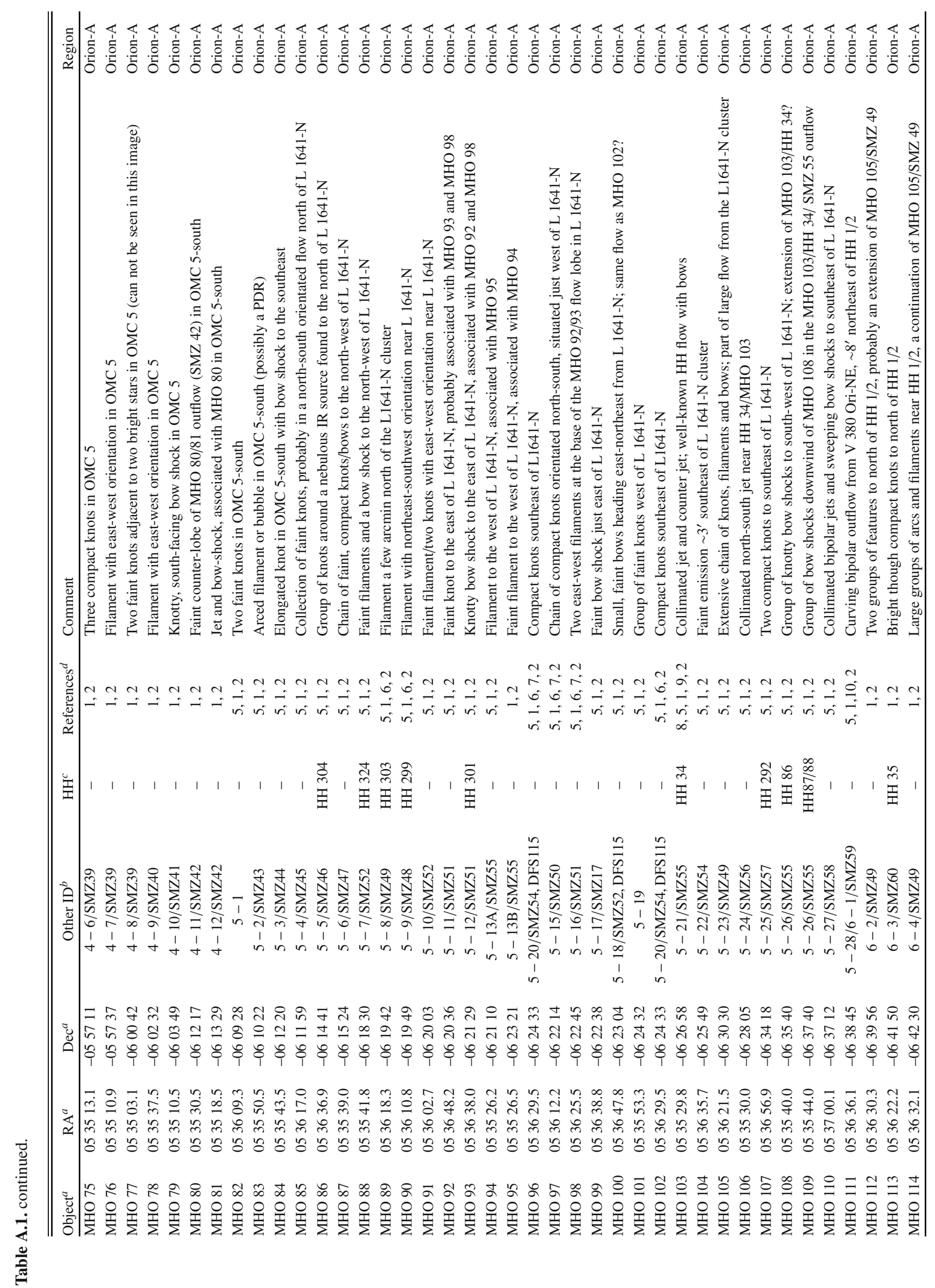



亦

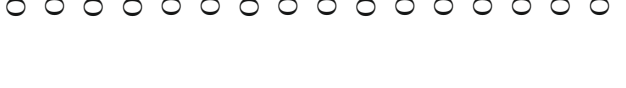

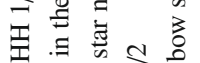

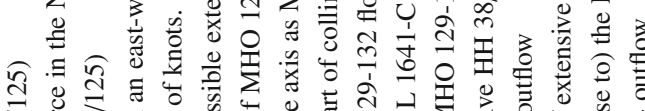

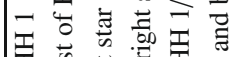

至

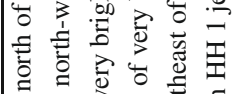

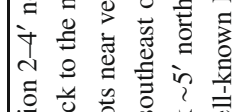

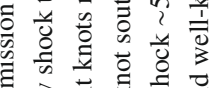

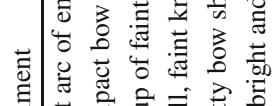

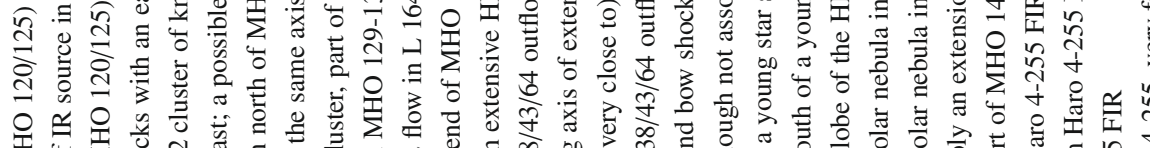

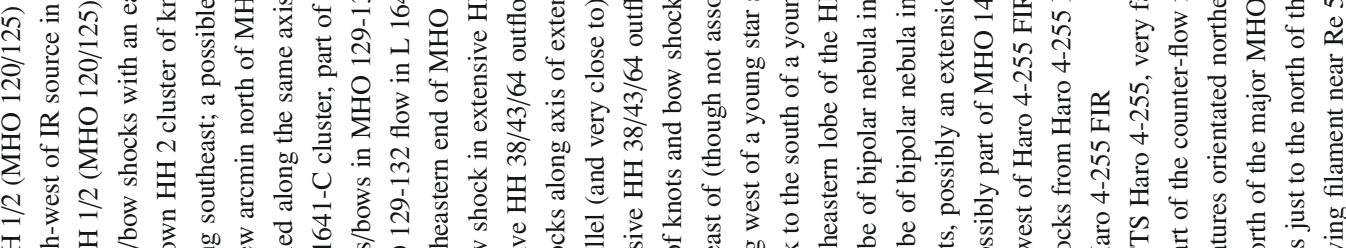

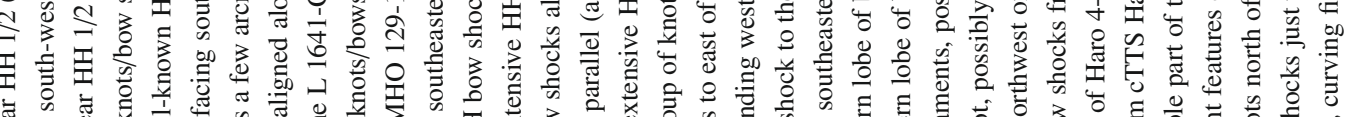

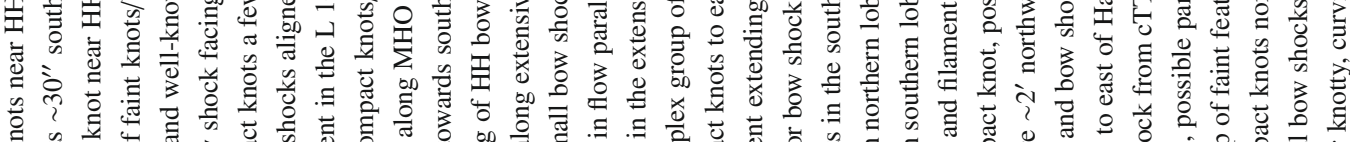

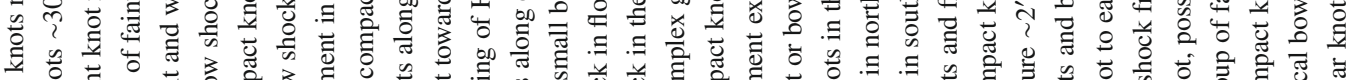

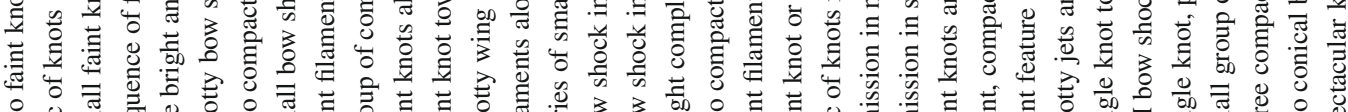

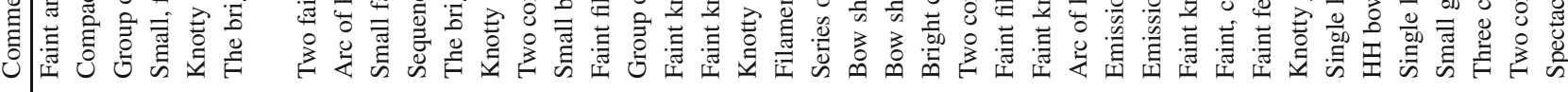

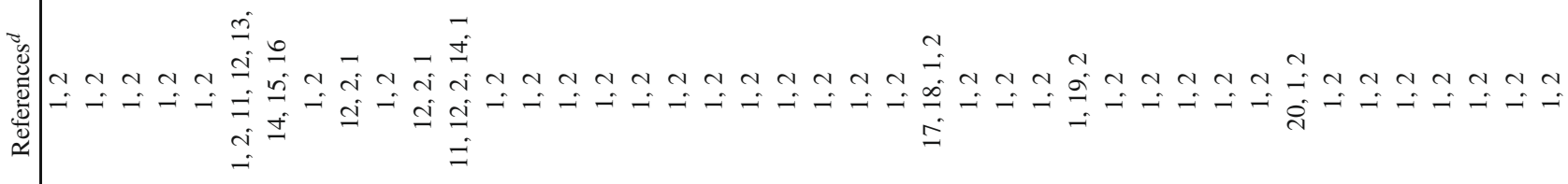

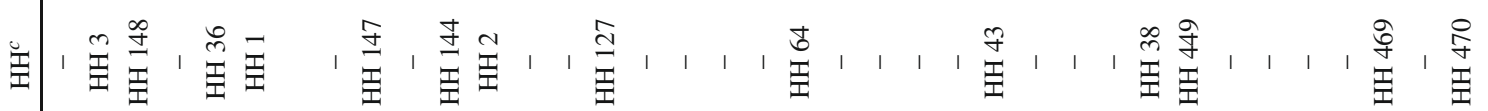

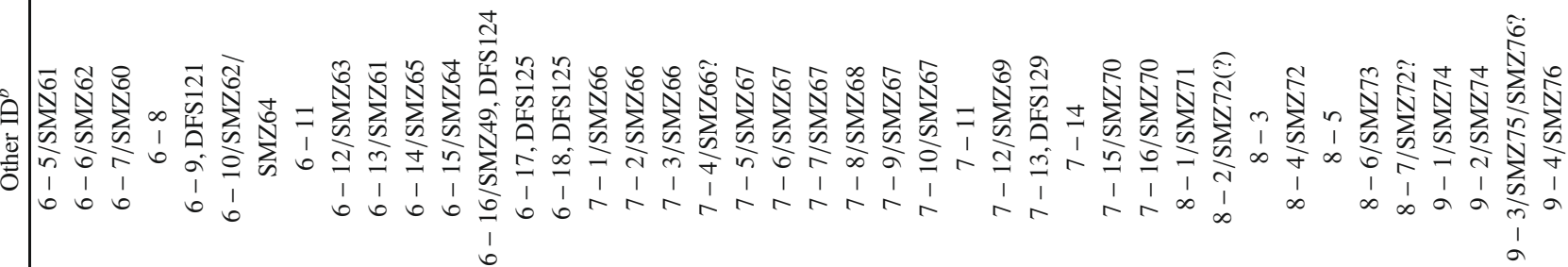

オป

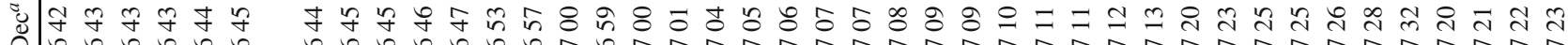
ص

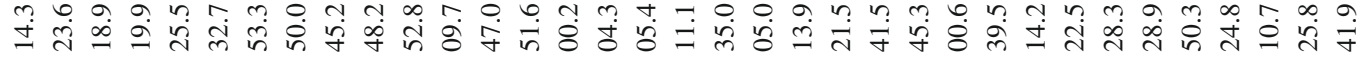
m

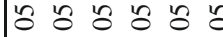

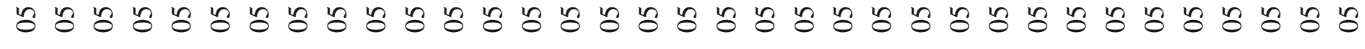

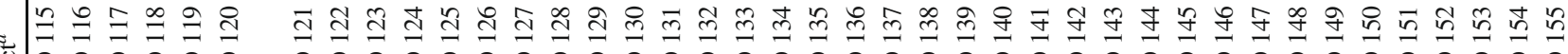

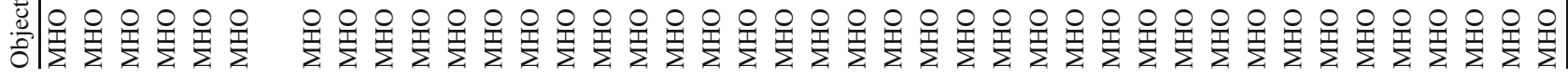




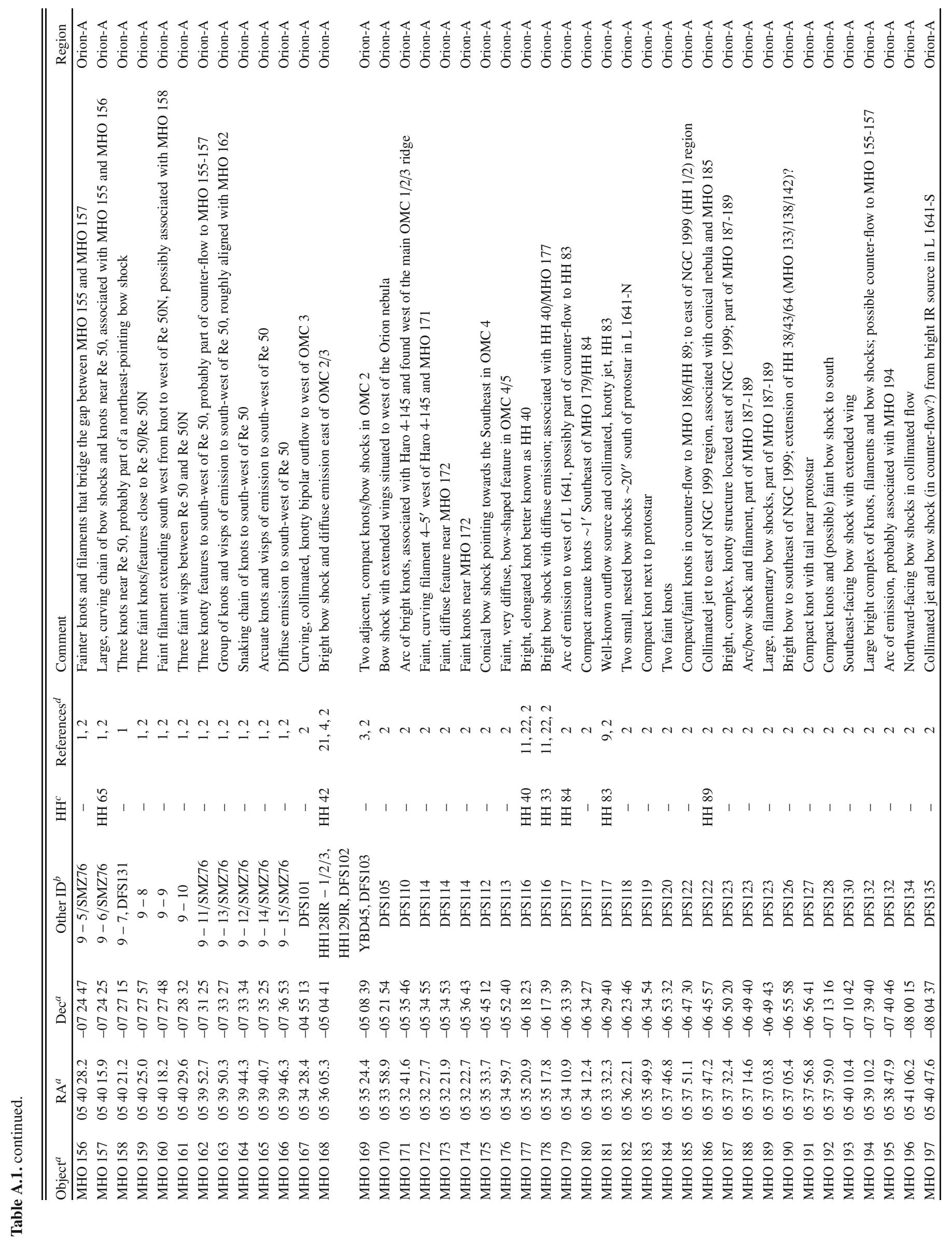

Page 12 of 36 


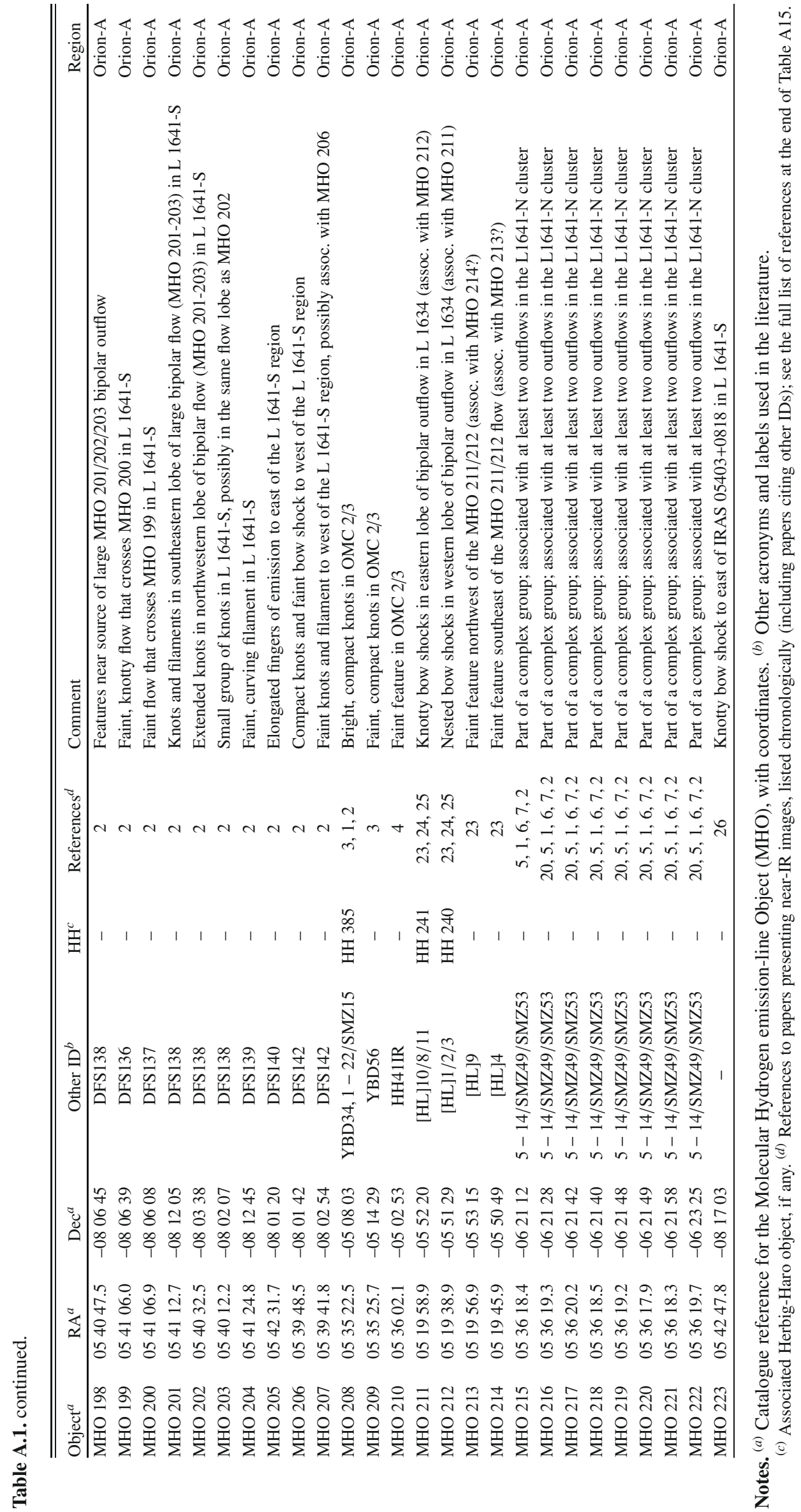




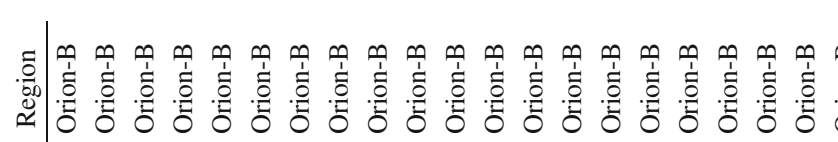

mp

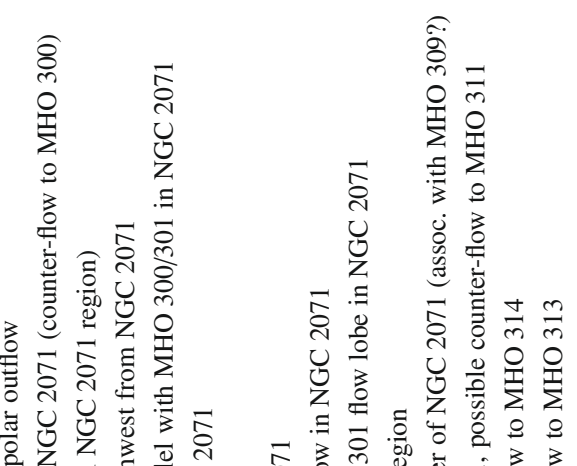

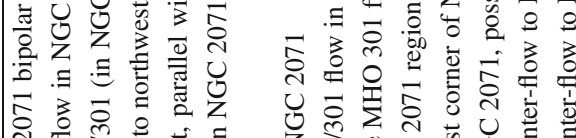

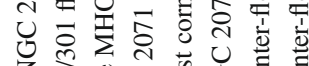

U

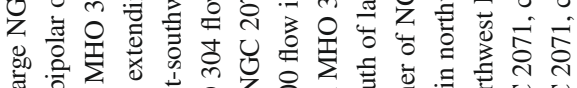

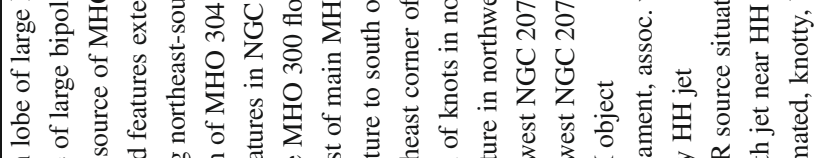

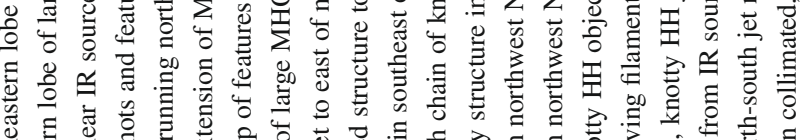

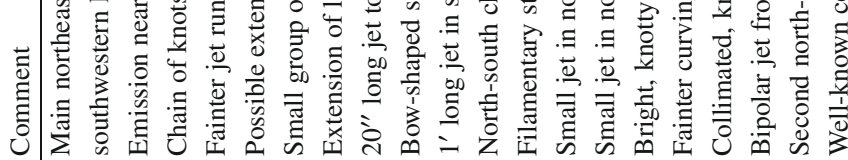



在市等

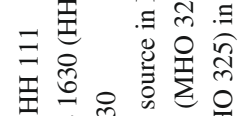

I.

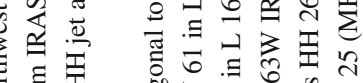

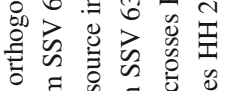

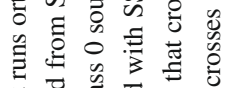

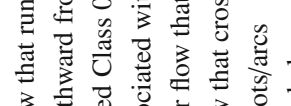

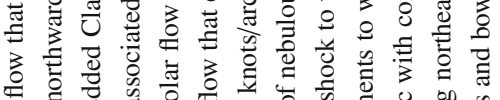

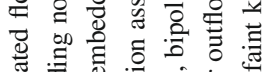

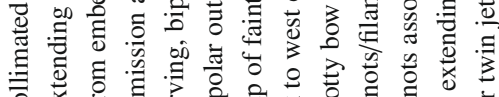

亏ั0

Int

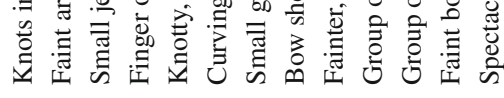

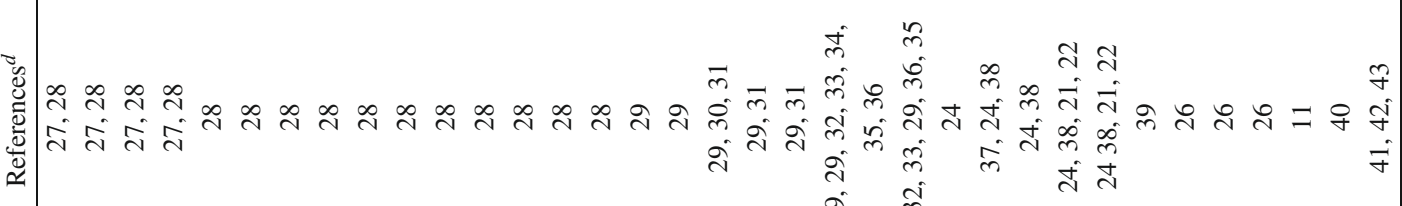



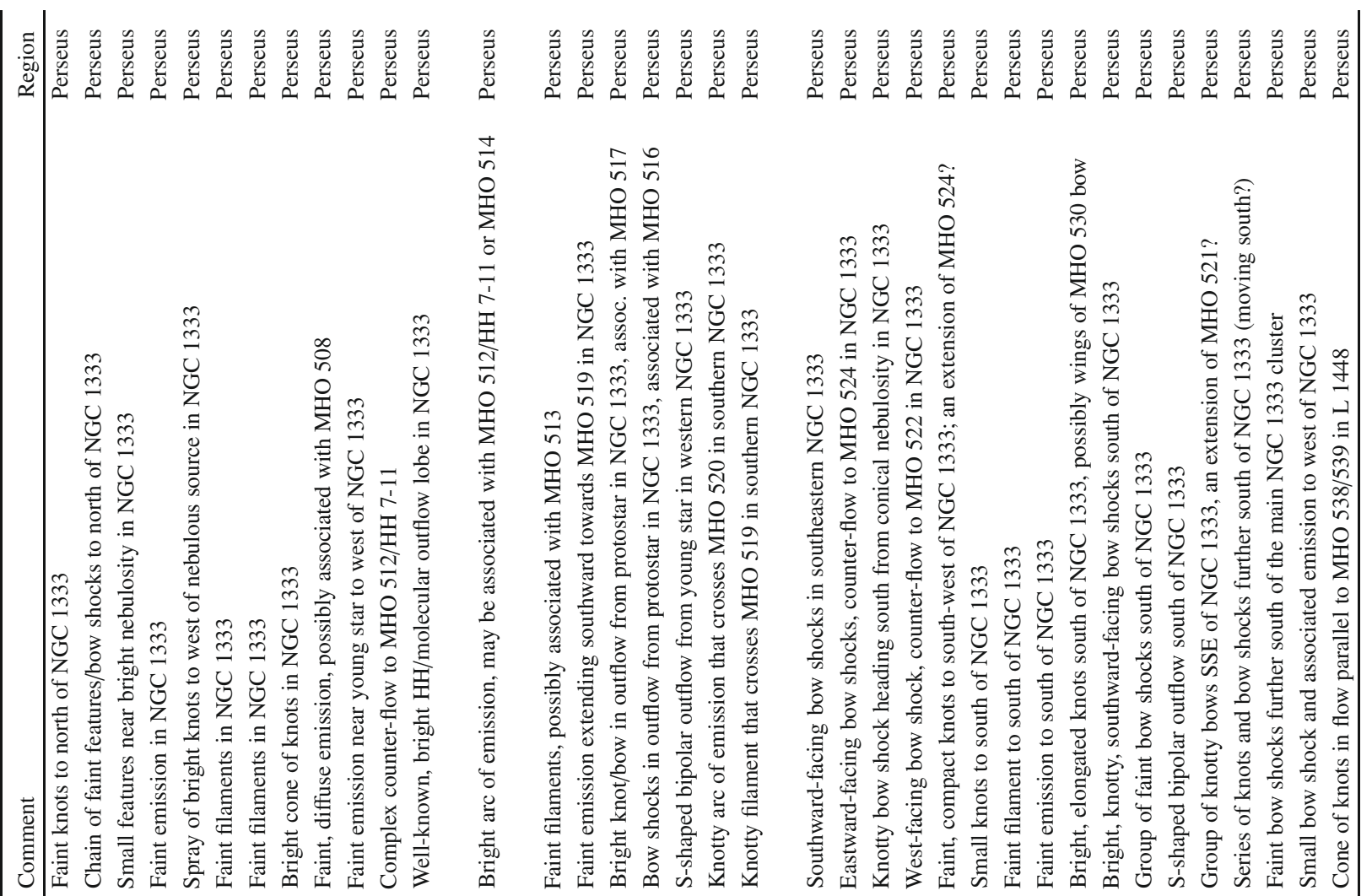

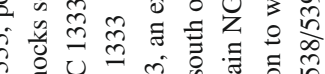

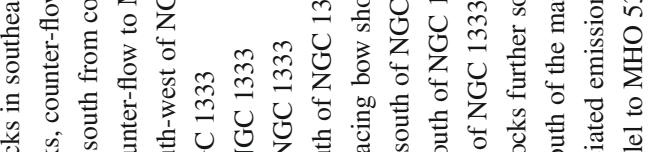

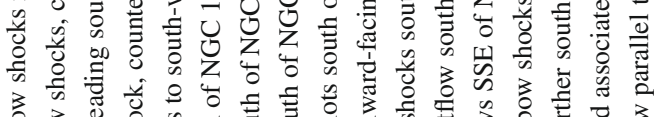

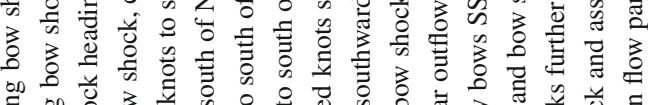

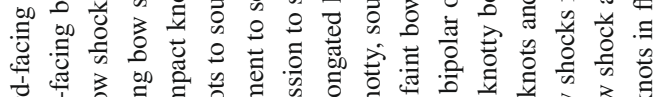

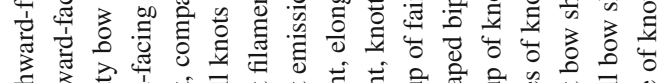

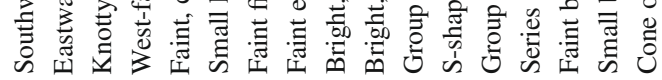
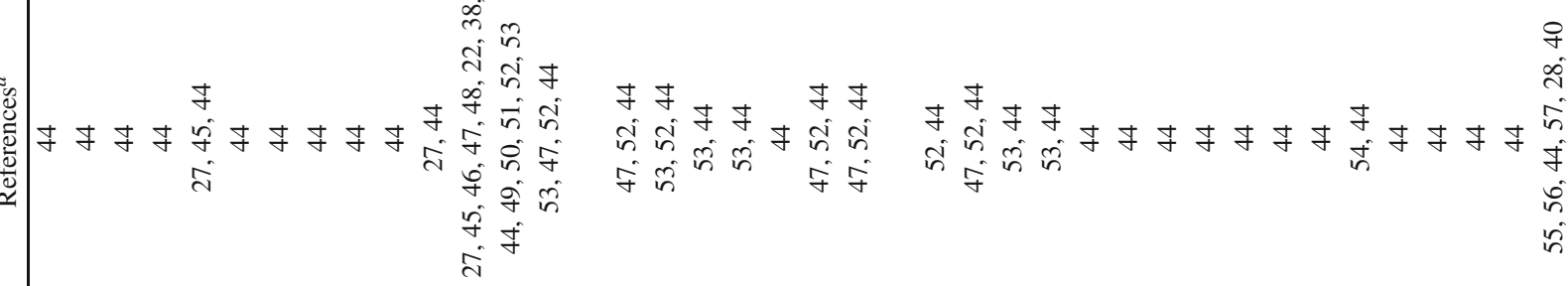

| 芺

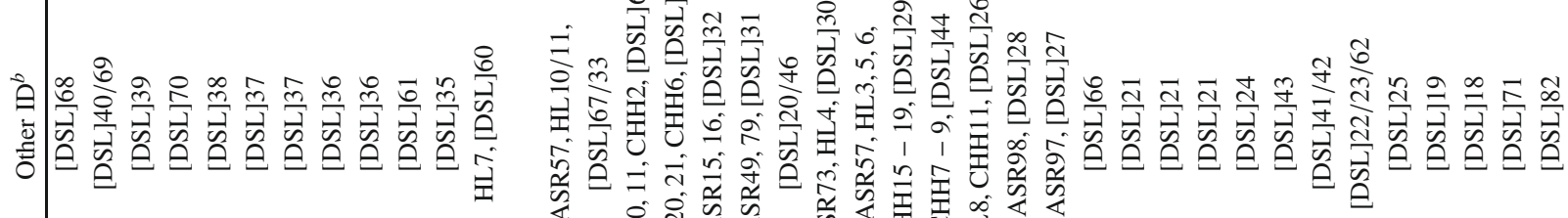

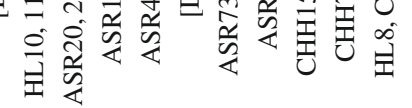

กิ ₹

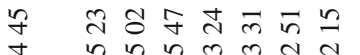

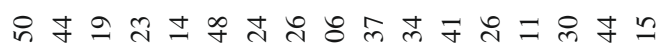

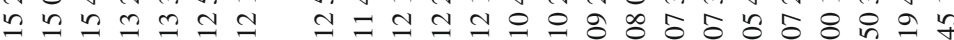

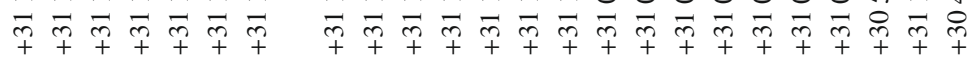

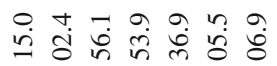
芒

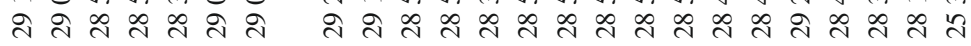

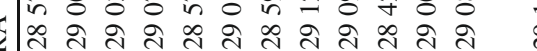

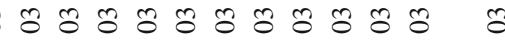

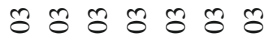

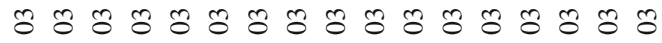

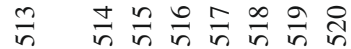

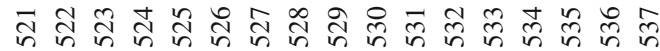

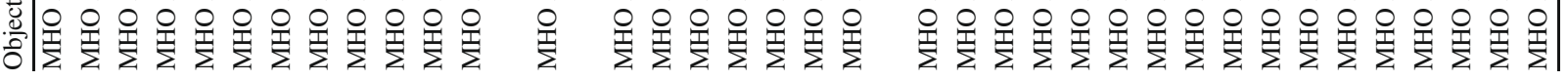




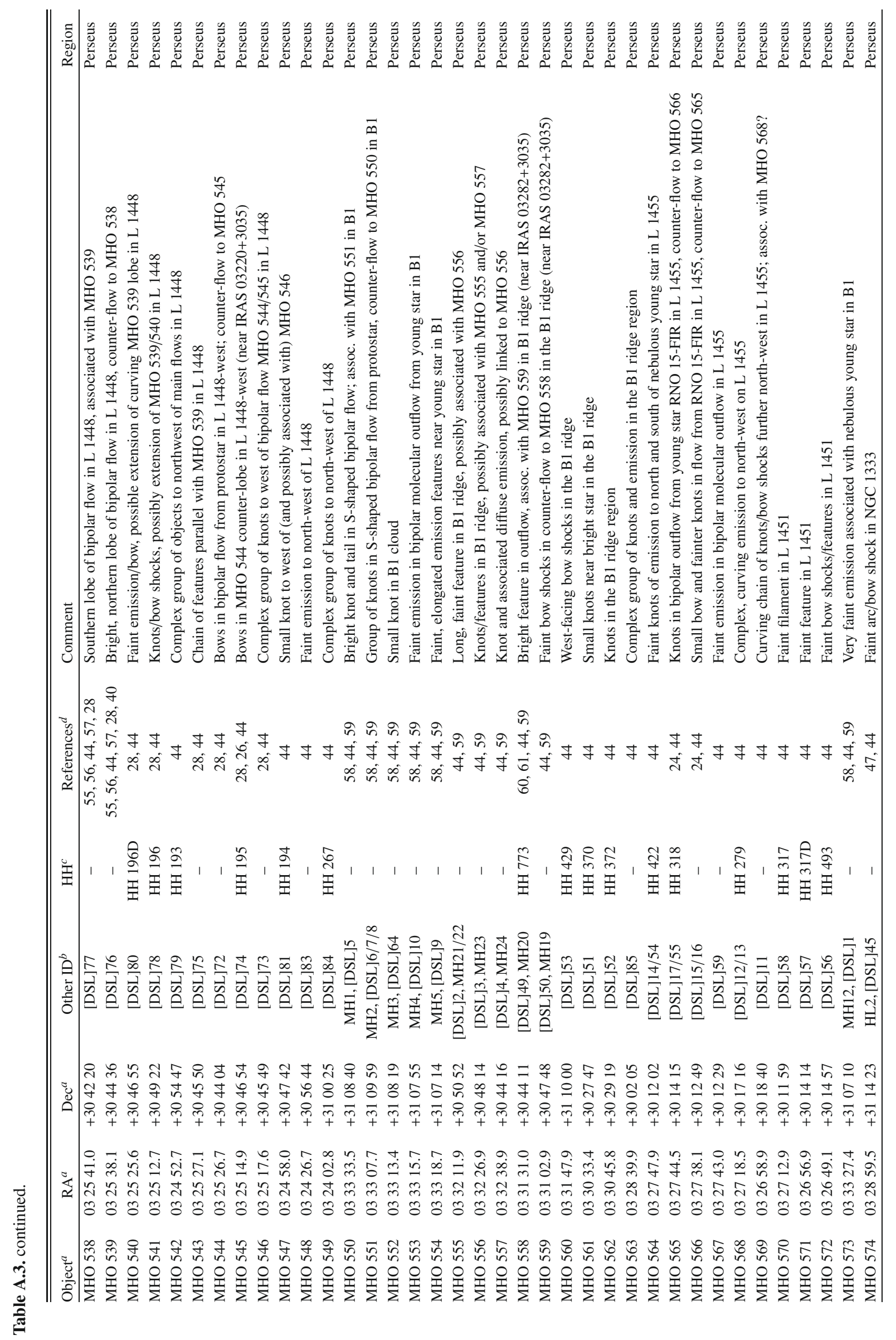




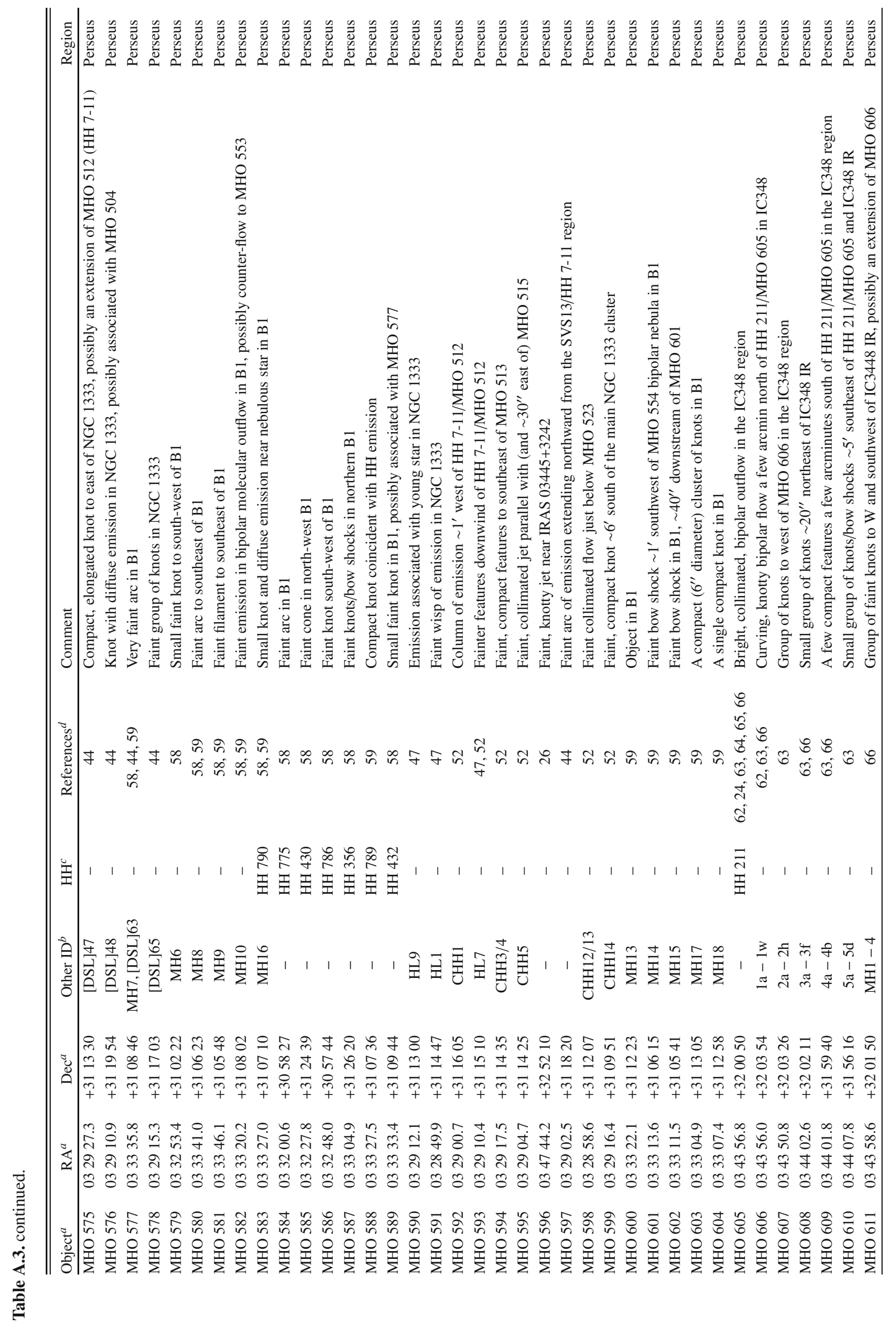




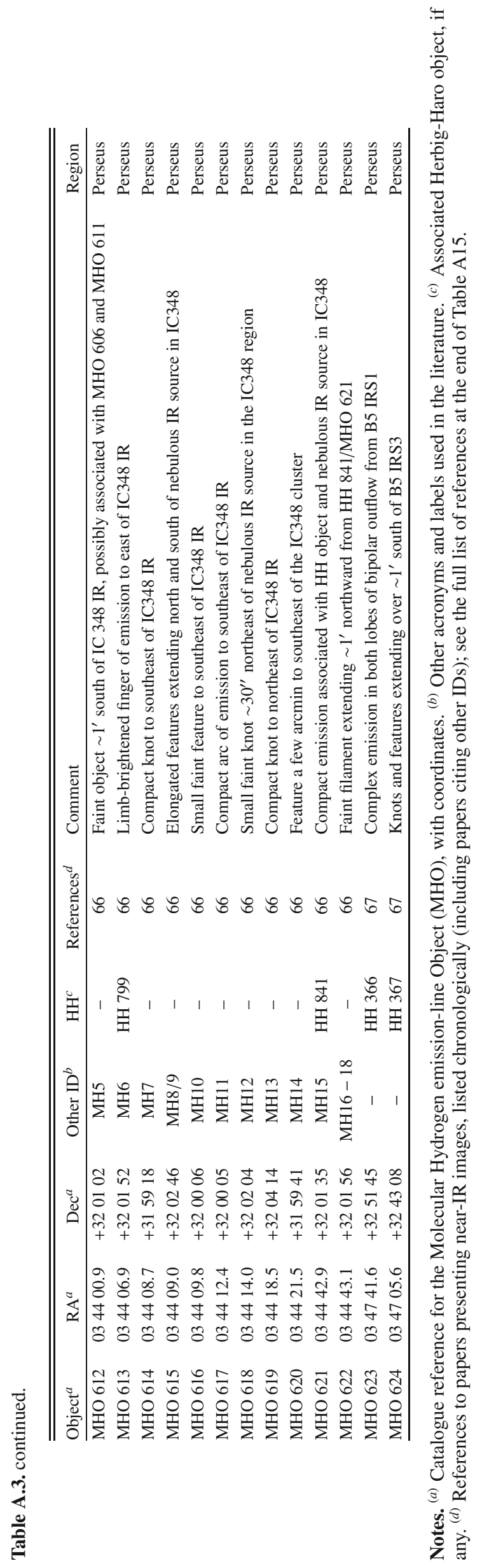

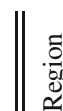

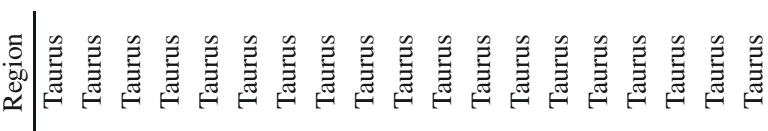
旁

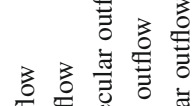

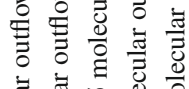

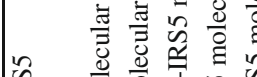

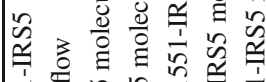
究言 昰

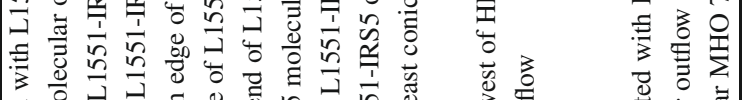

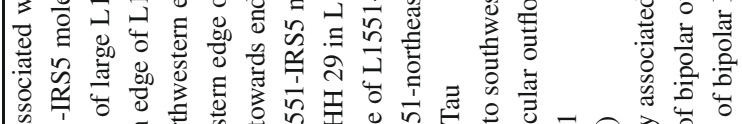

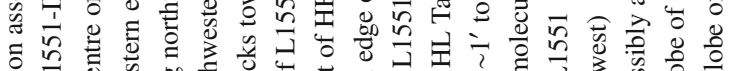

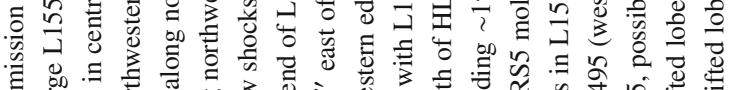

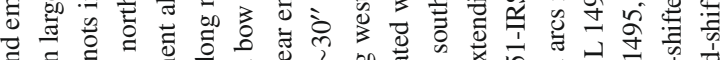

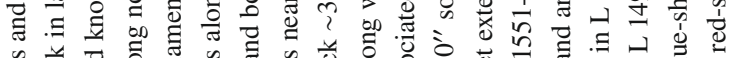

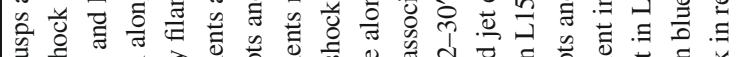

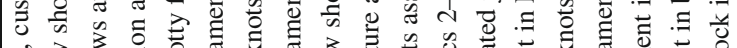

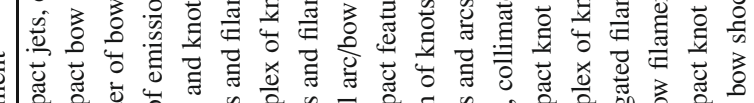

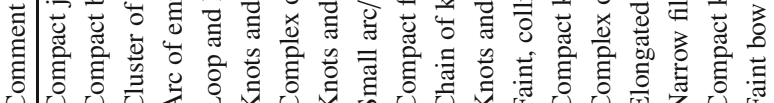

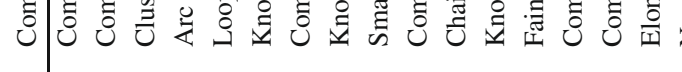
. $?$

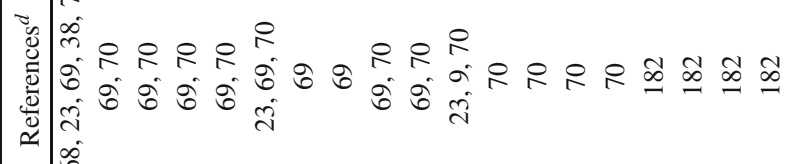

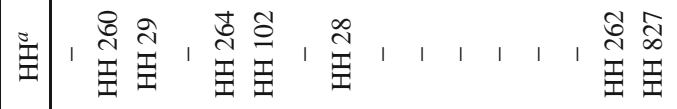

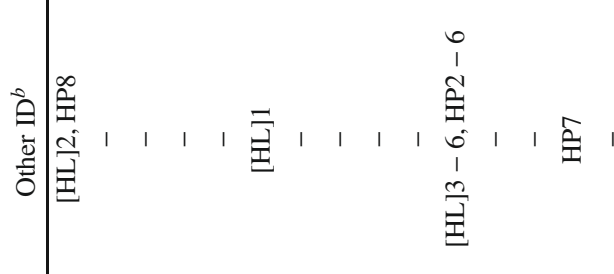

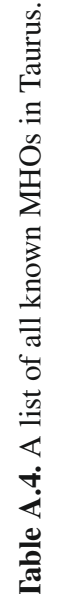

d

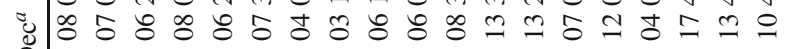

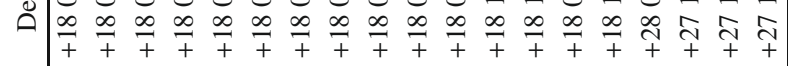

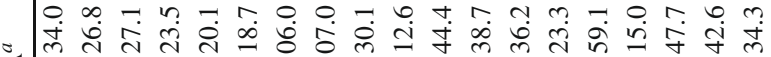
«্লা

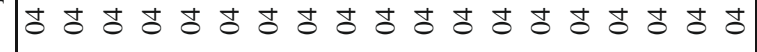

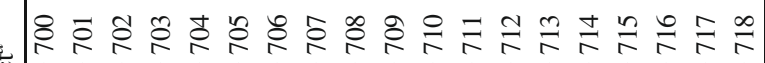

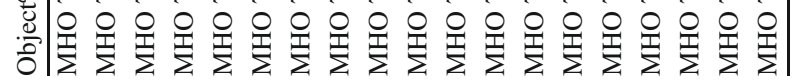




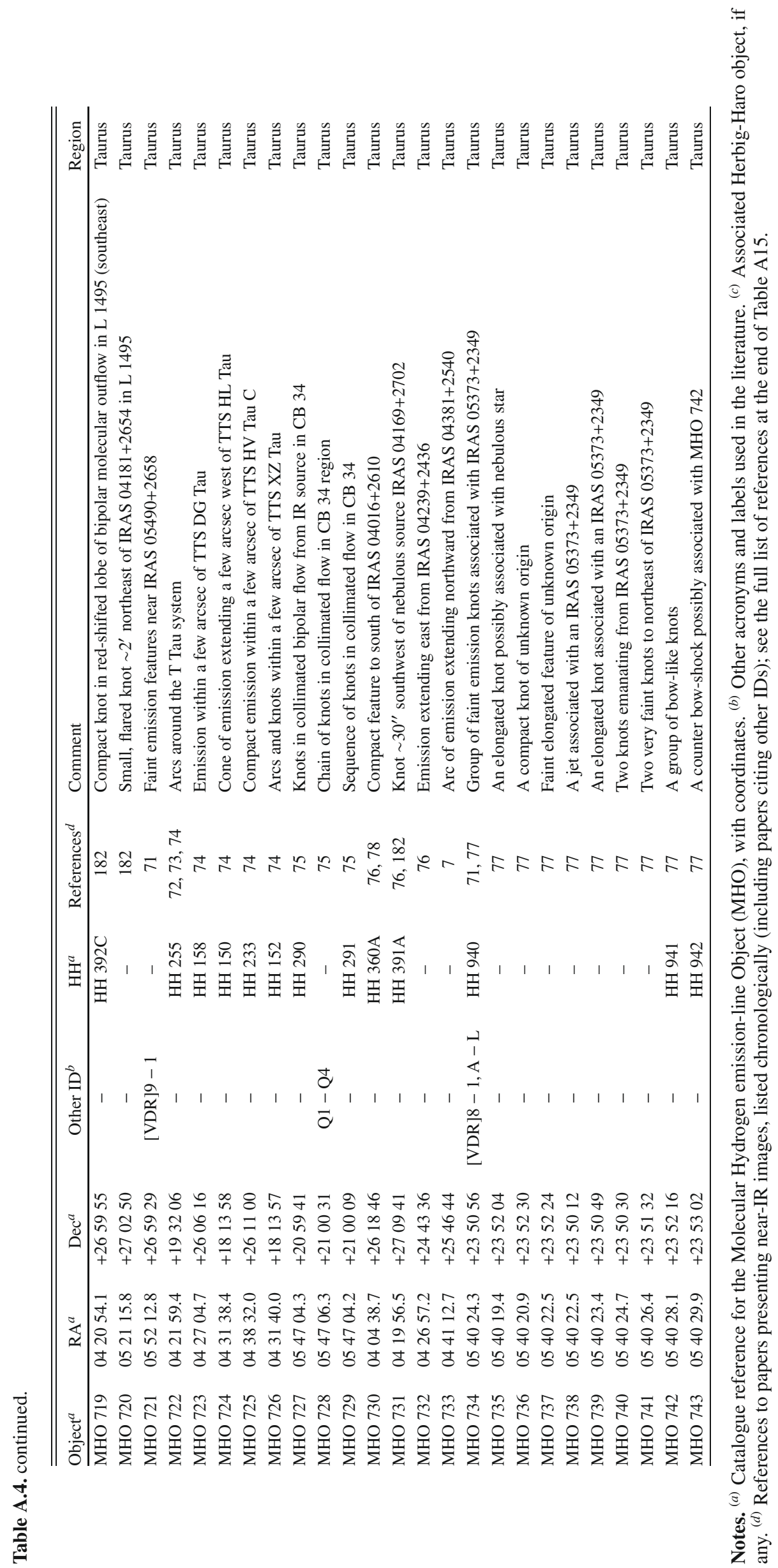




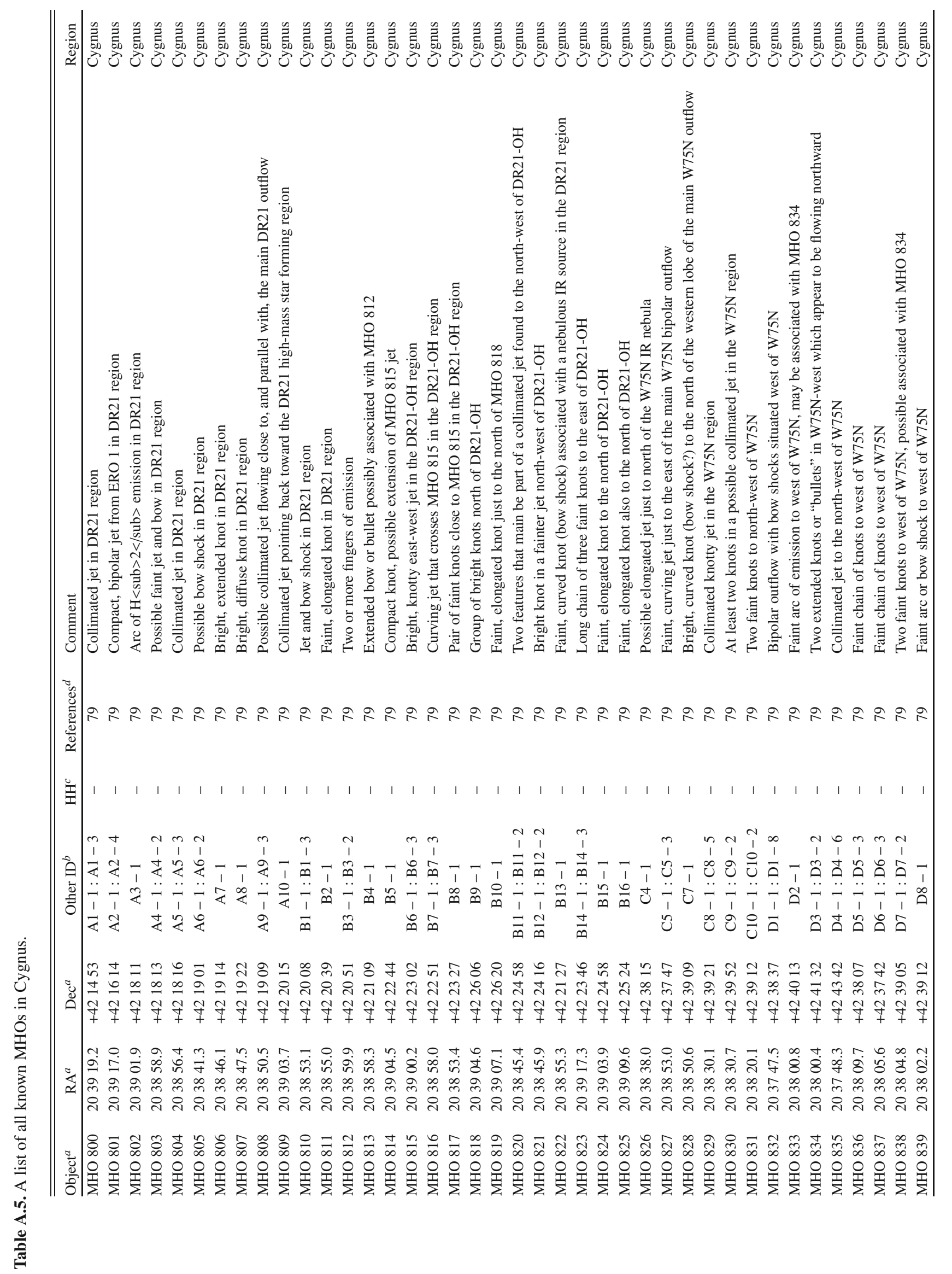




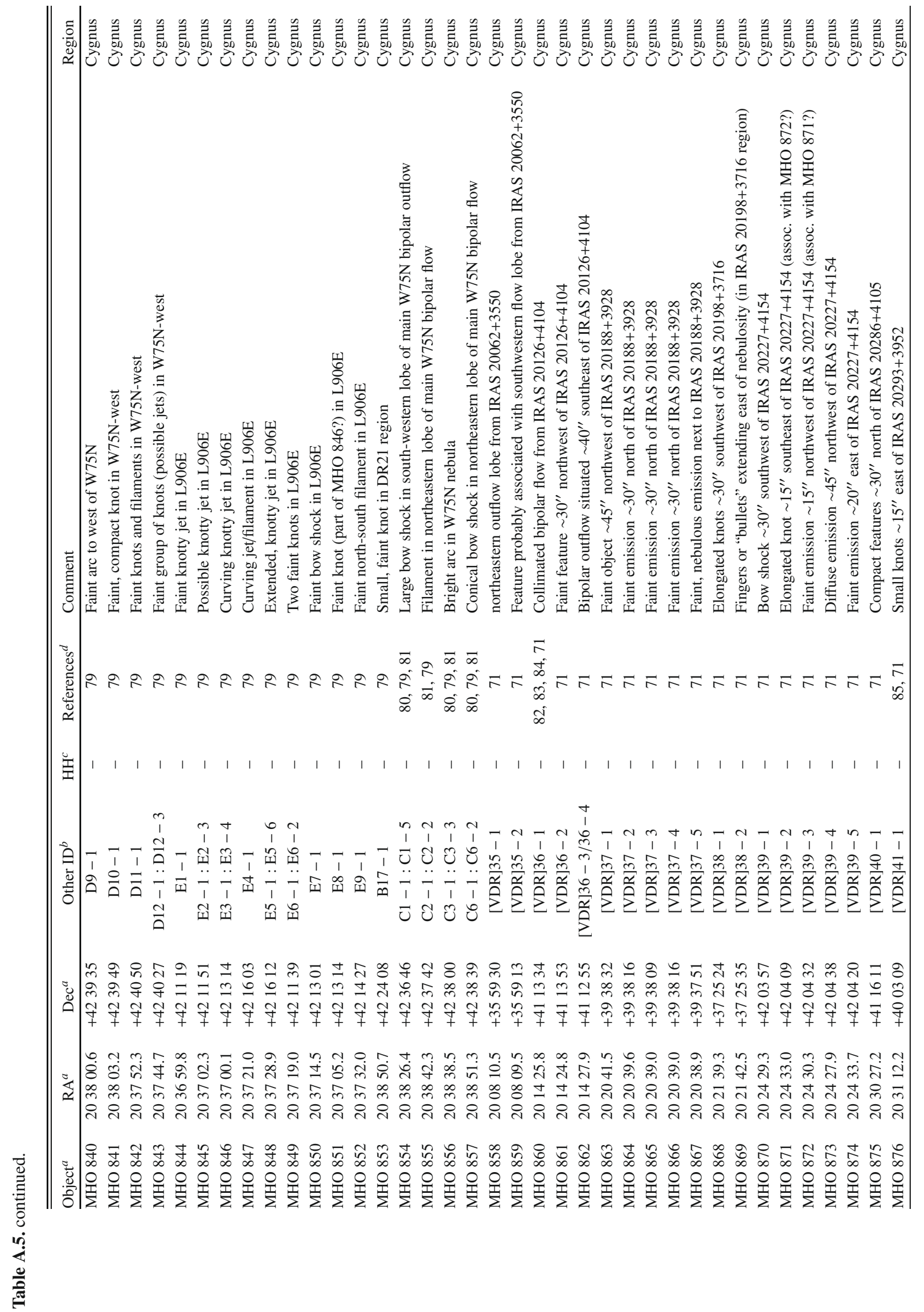




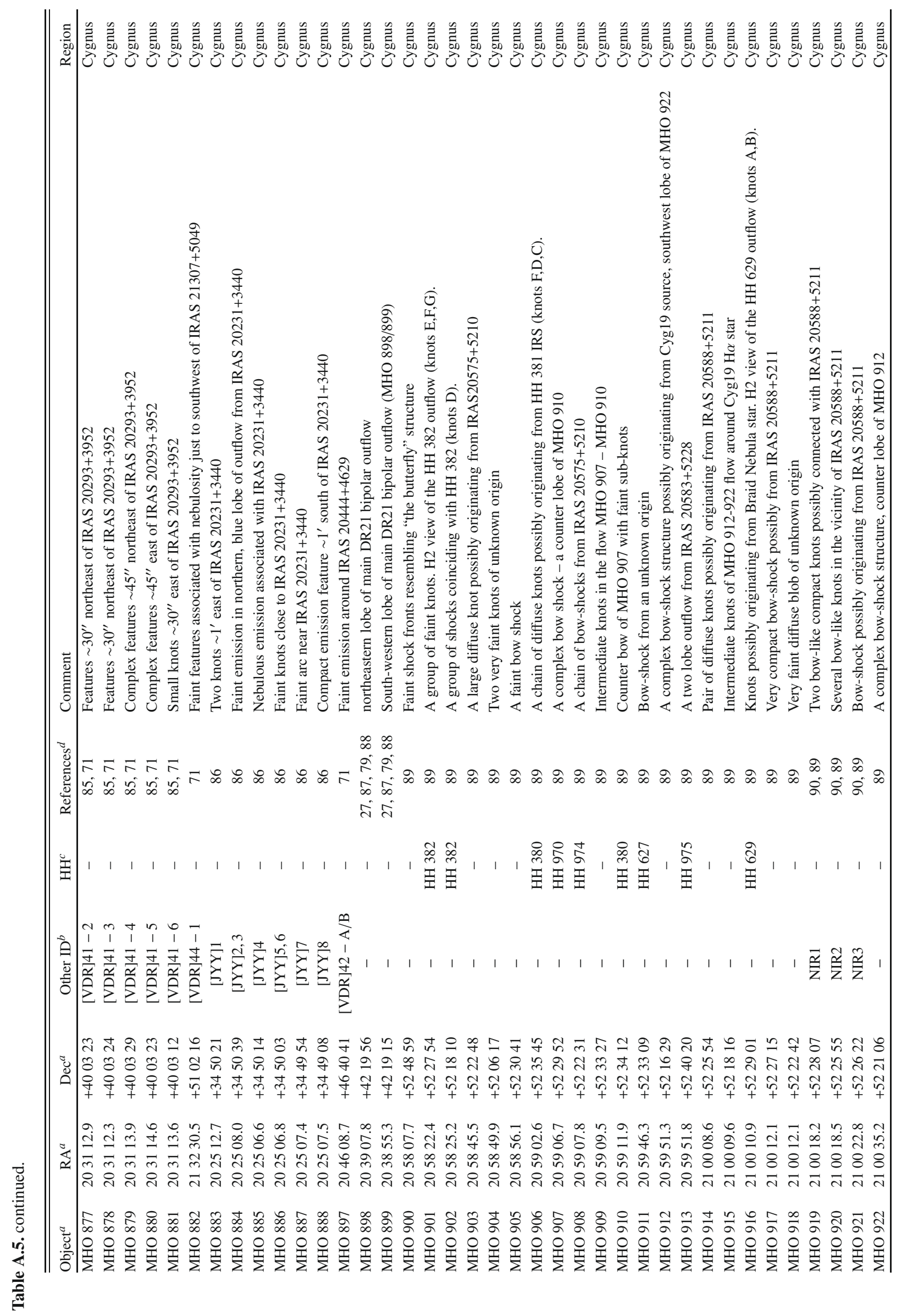




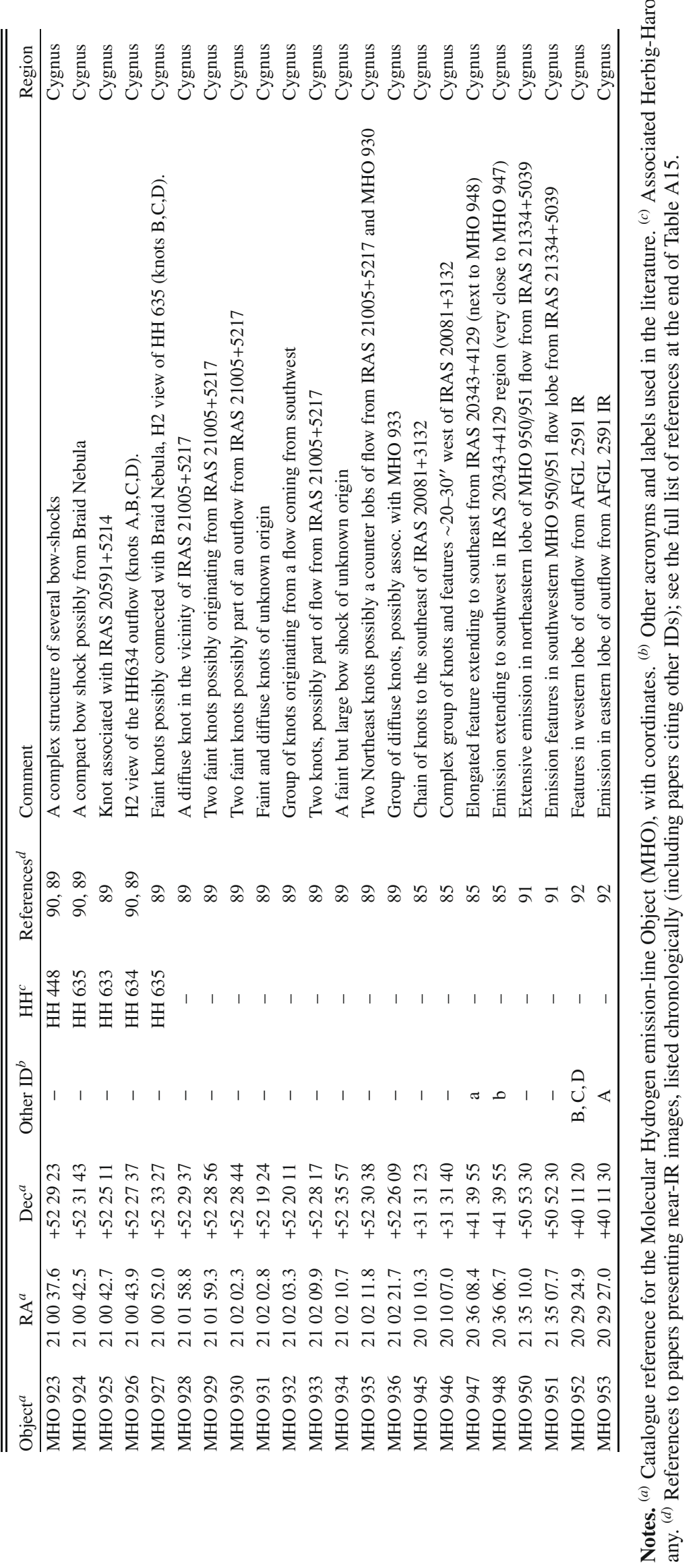




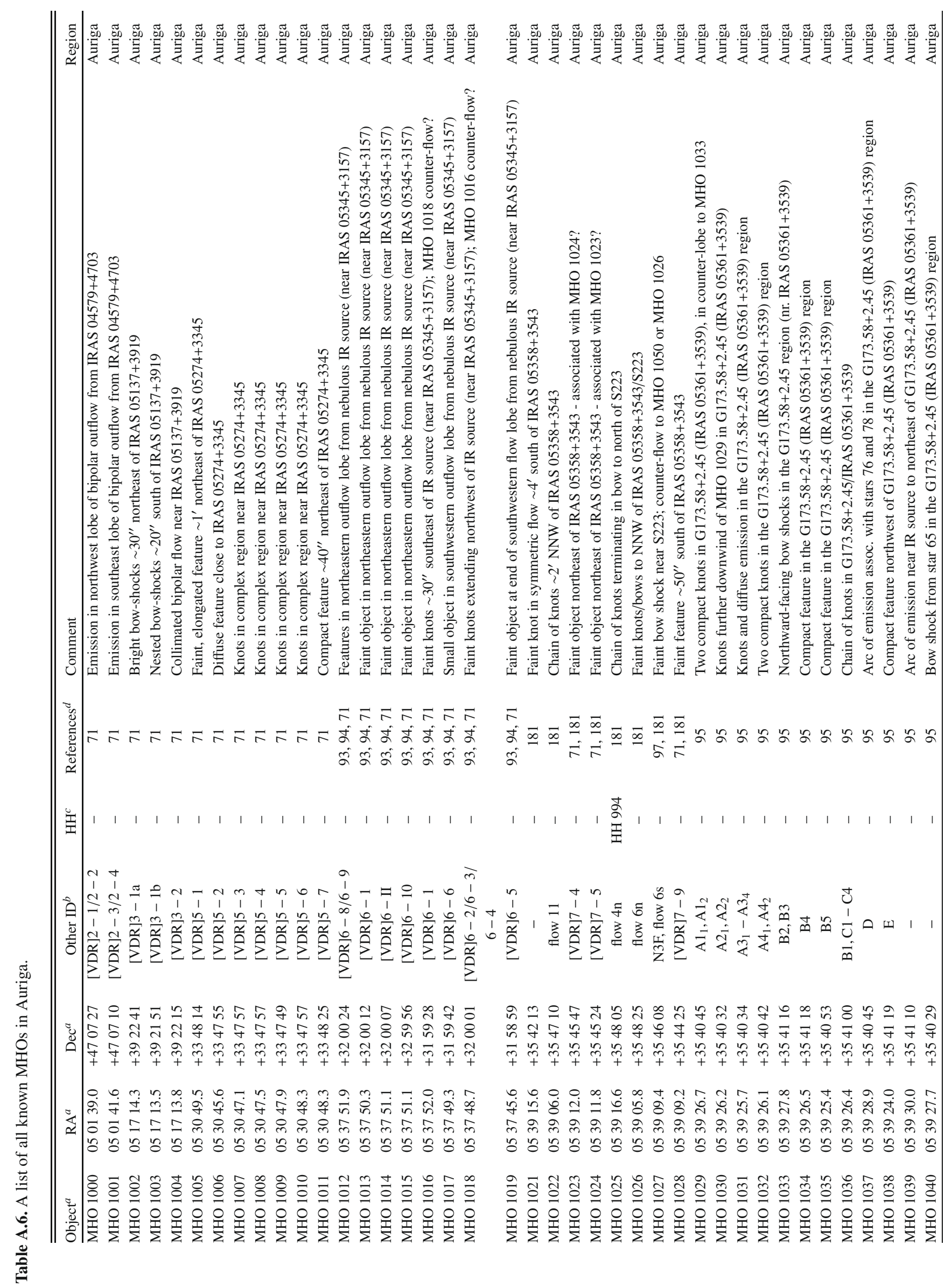


C. J. Davis et al.: A general catalogue of molecular hydrogen emission-line objects (MHOs) in outflows from young stars

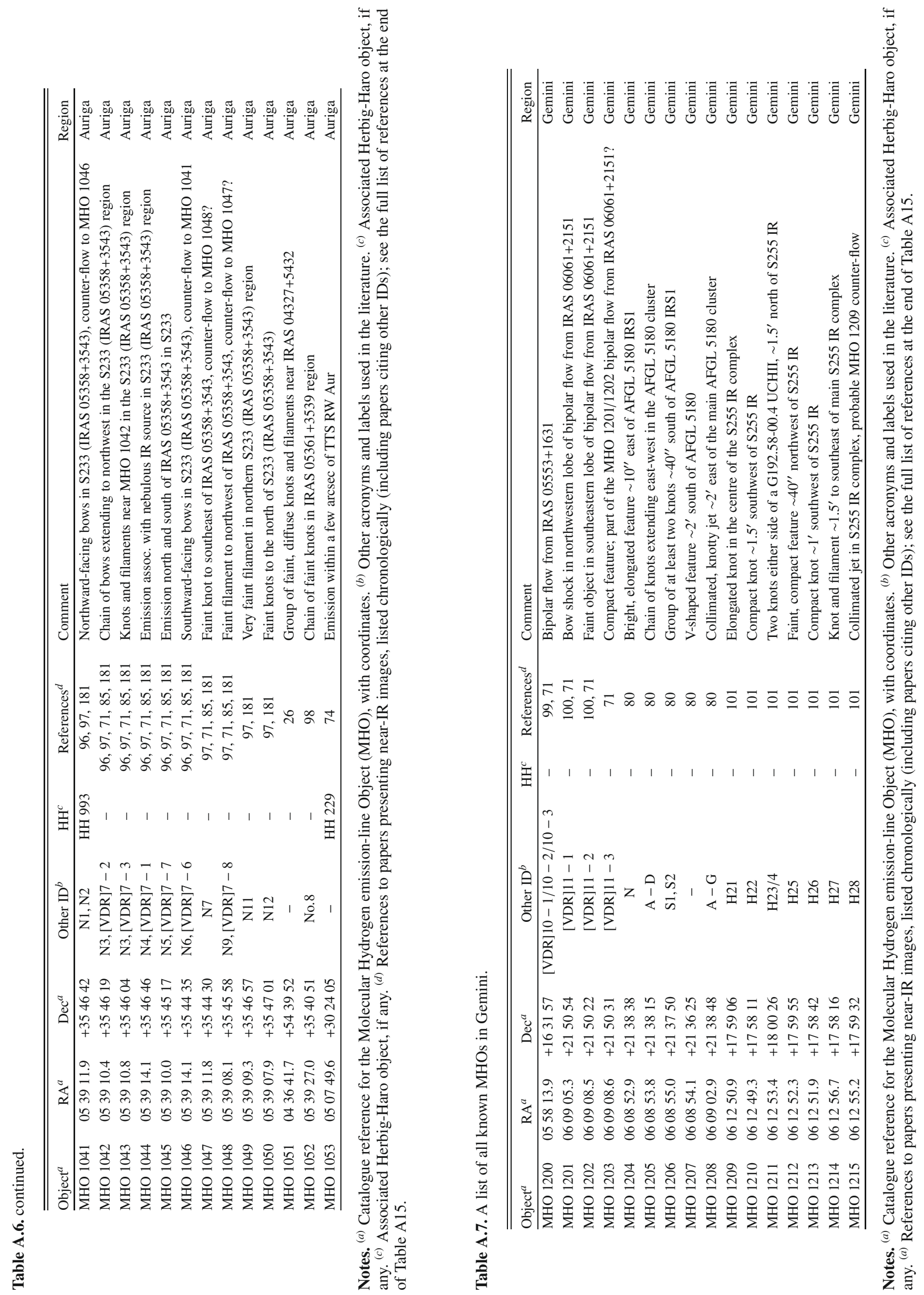




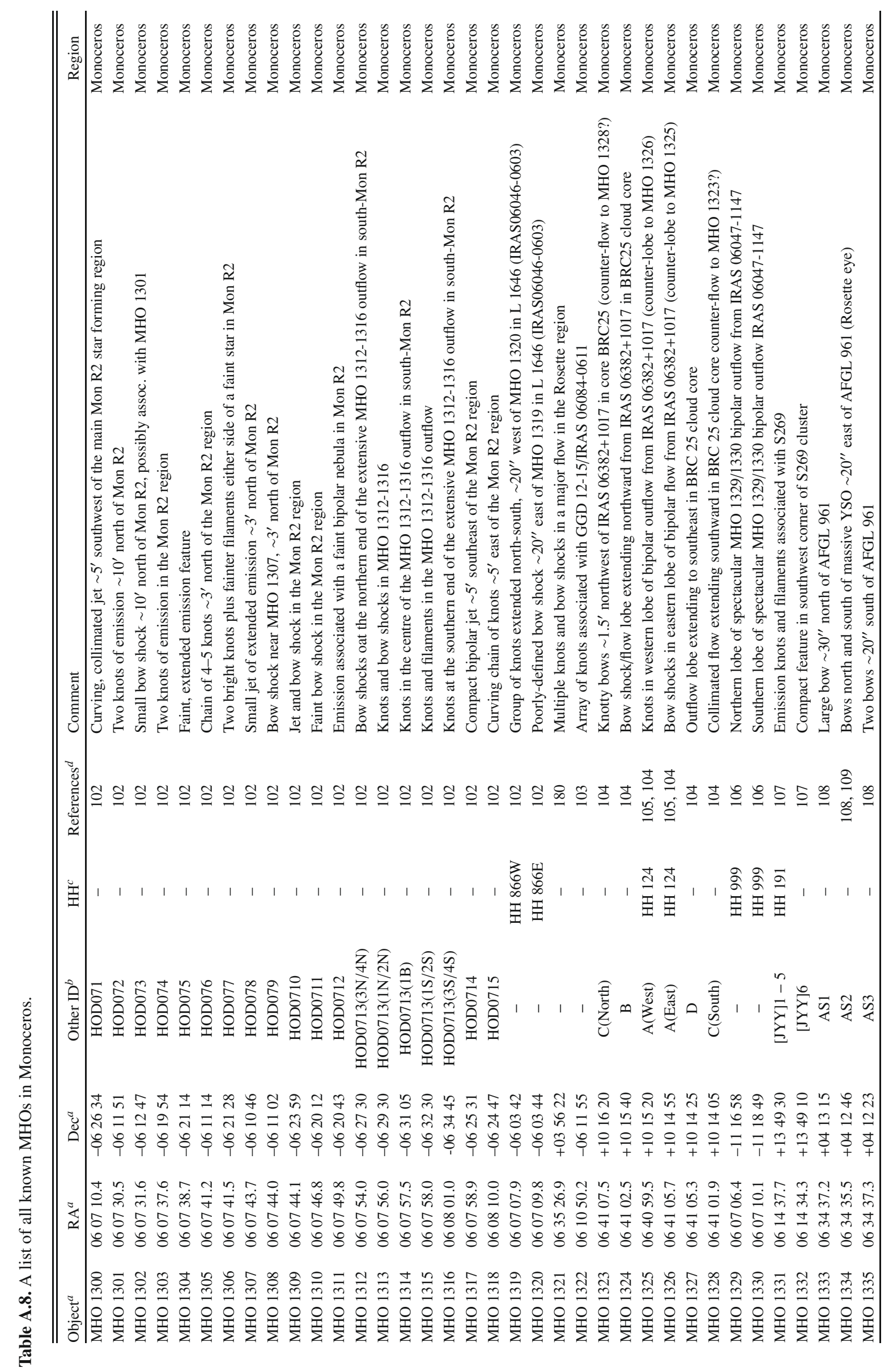




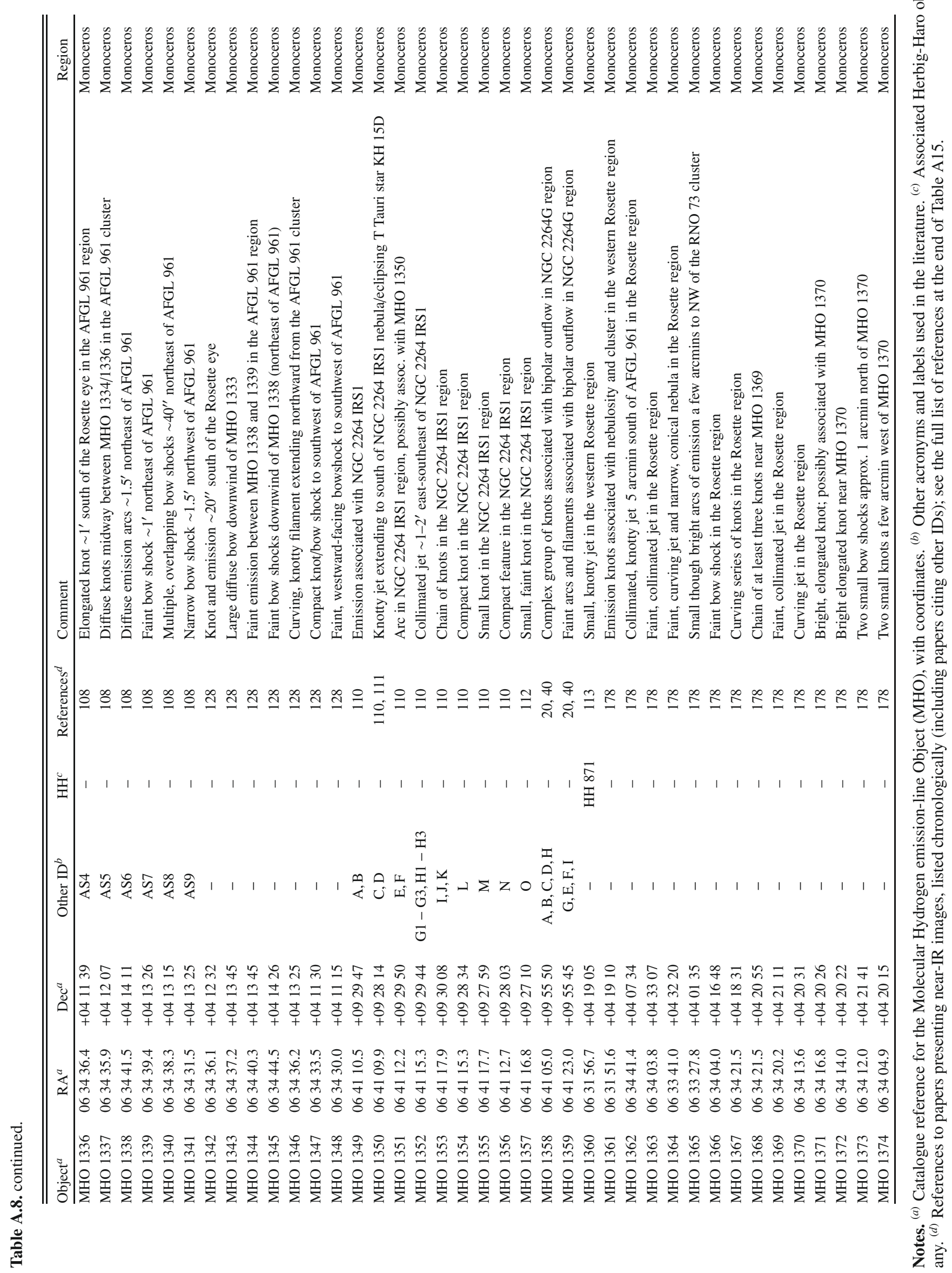




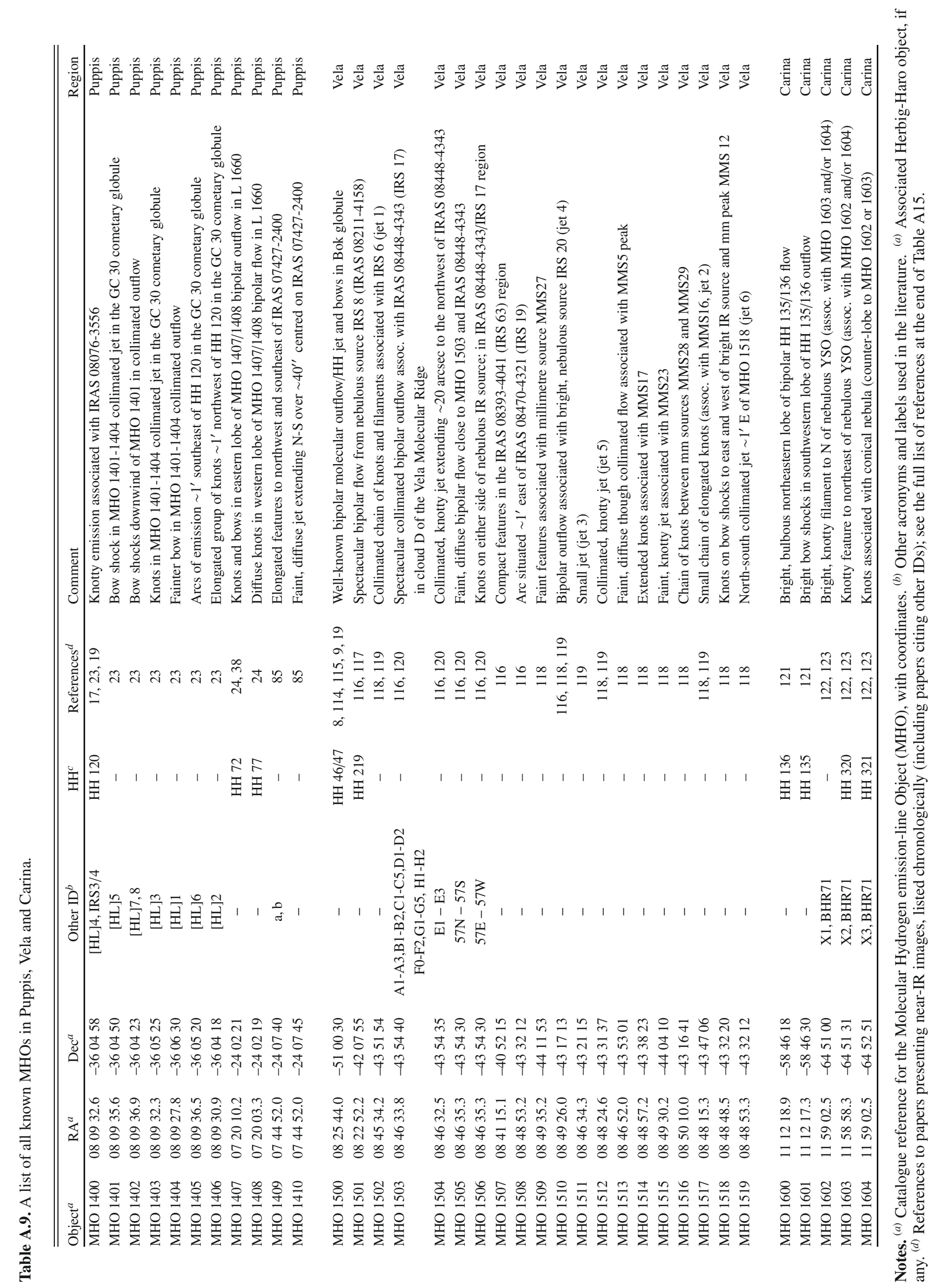




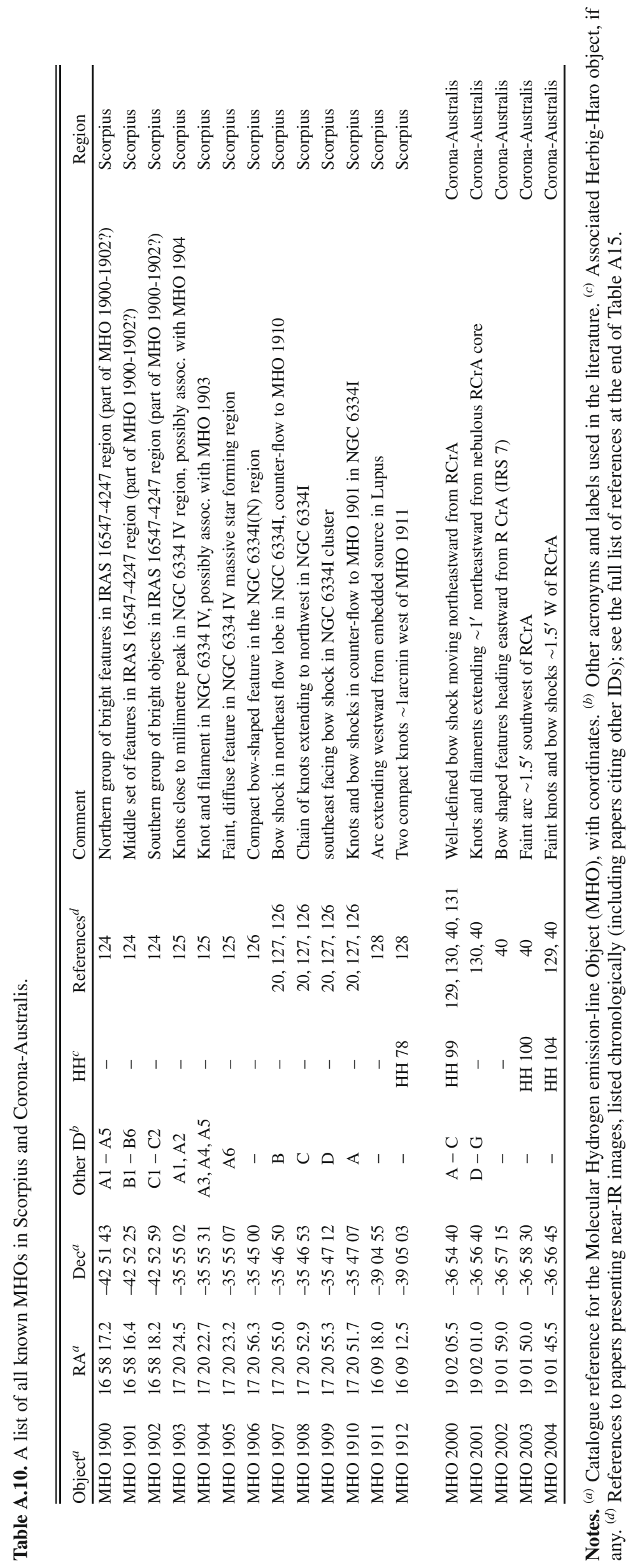




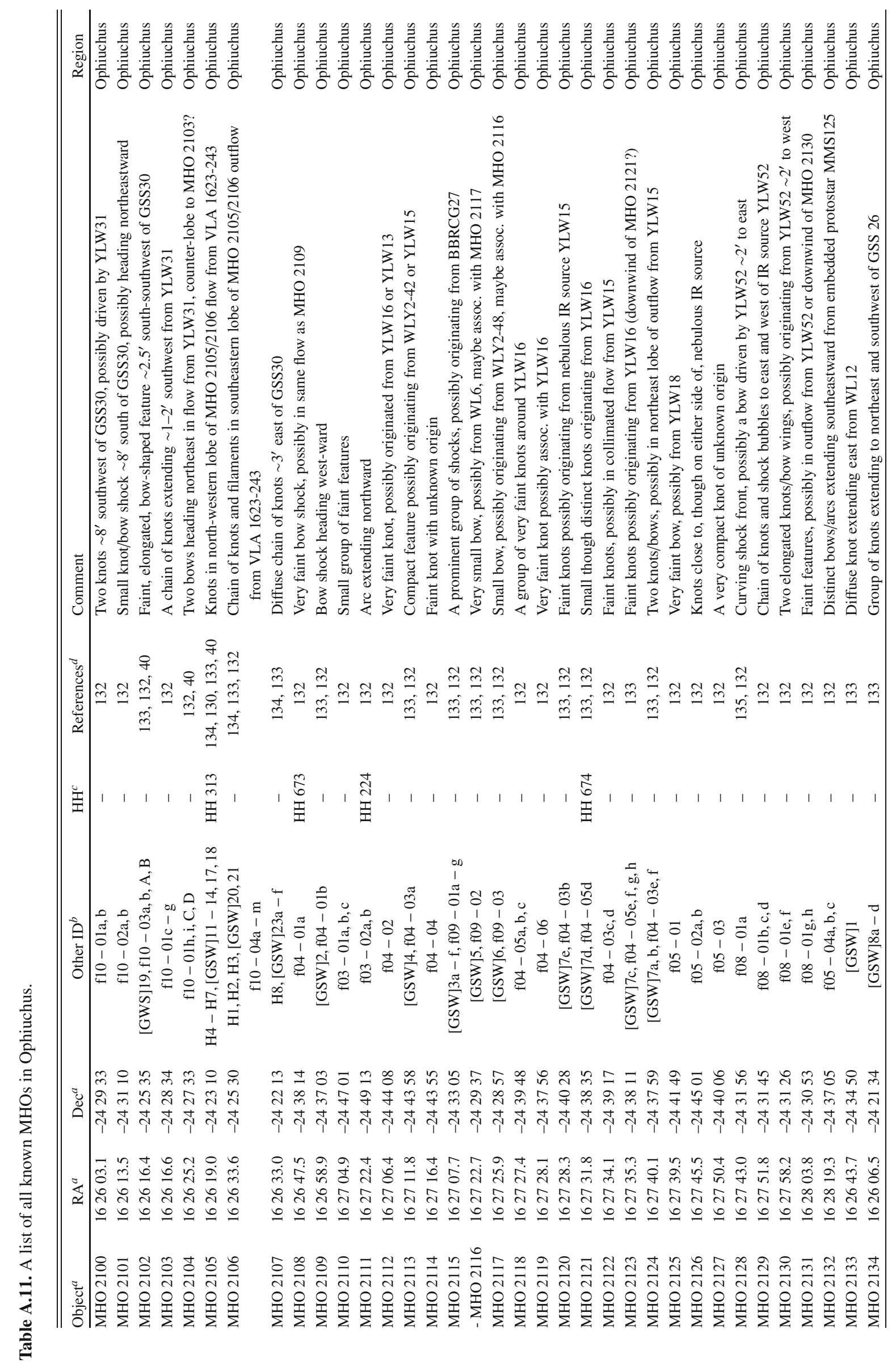


C. J. Davis et al.: A general catalogue of molecular hydrogen emission-line objects (MHOs) in outflows from young stars

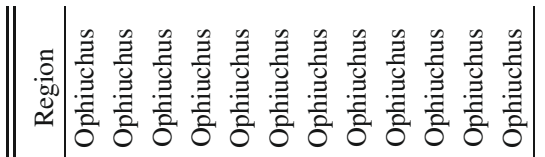

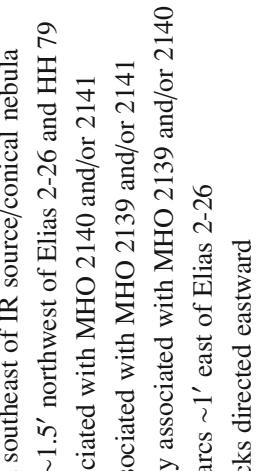

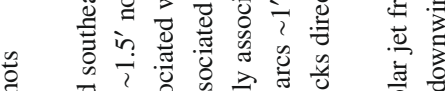

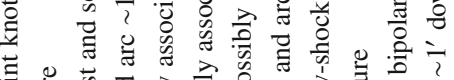

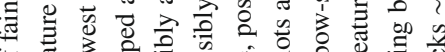

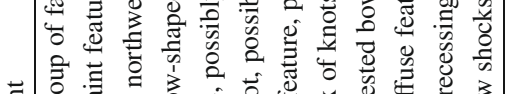

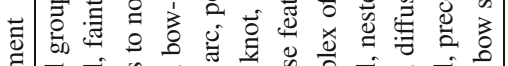

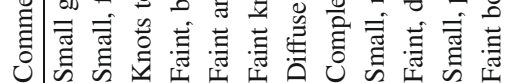
$=$

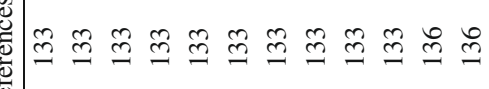
$\approx$

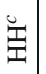

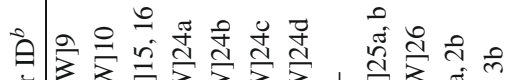

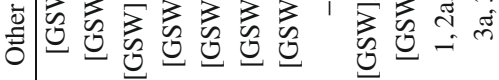
অ ¿

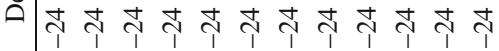
ก

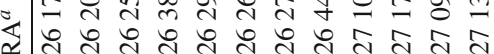

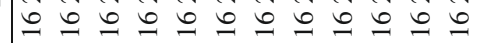
m t5)

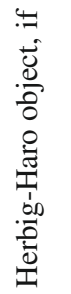

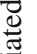

悹

e $\frac{0}{0}$

نㄹ

륜음

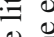

올

$\Xi \pi$

D

$\frac{n}{8}$

吾

है

월

एक

蒒

نे

总影

ठ․



$\sum \bar{\Xi}$

흥 강

웅

.0ํำ

है च

공

0
0
0
0
0
0

흘

造

要

훙

8
0
0
0
0

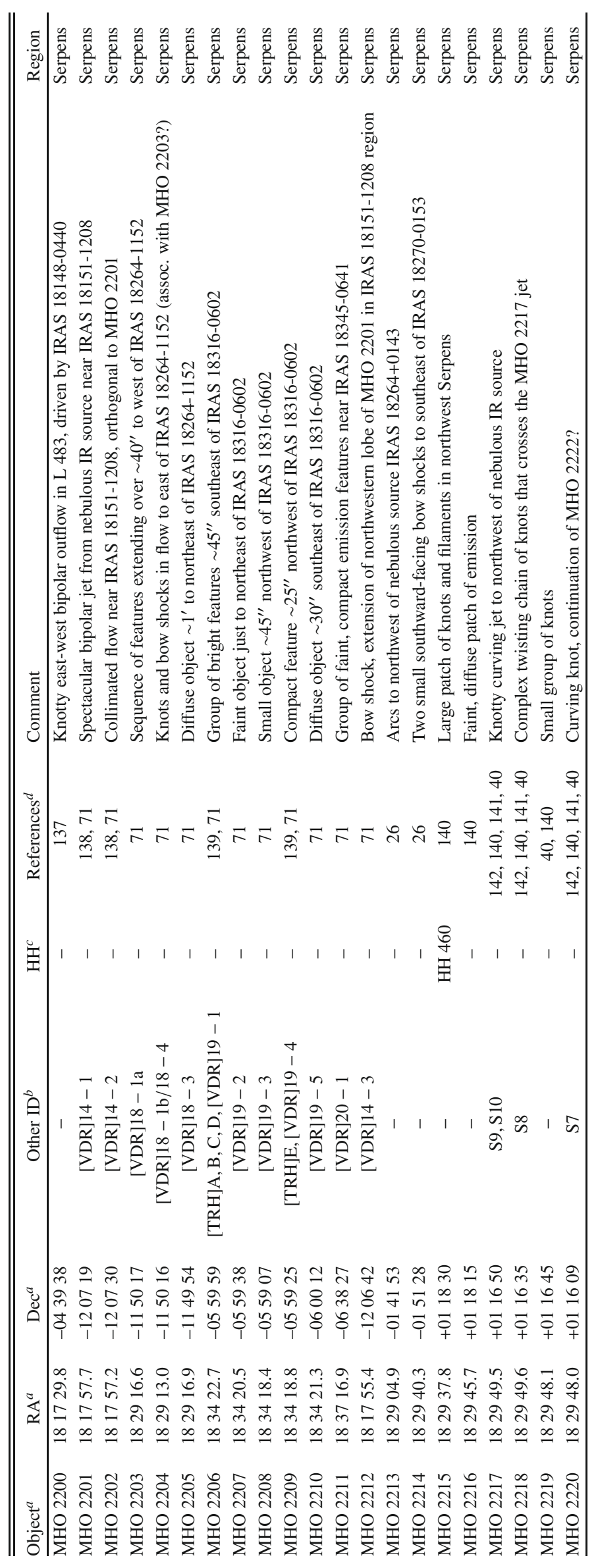

Page 31 of 36 







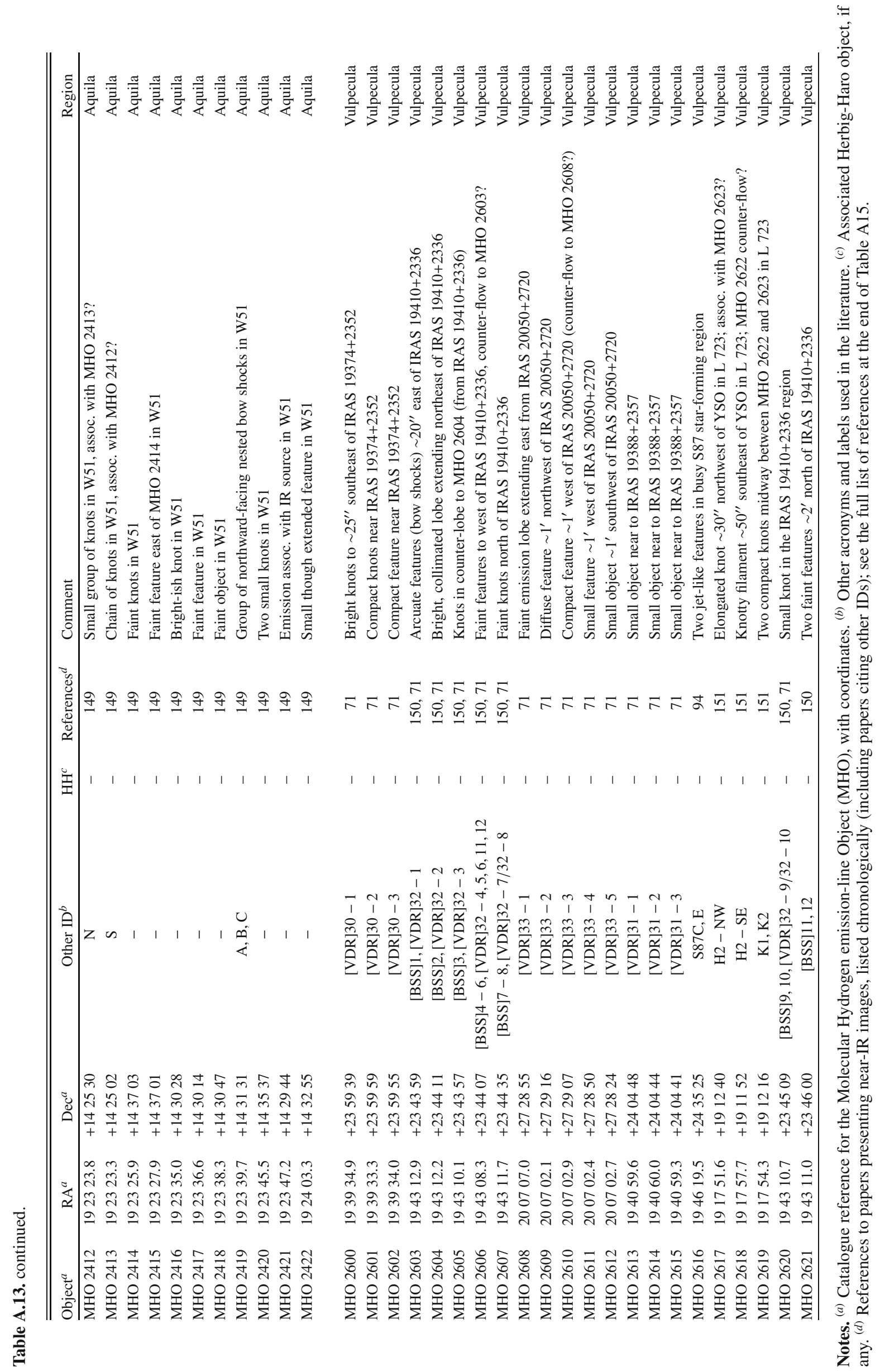

Page 33 of 36 


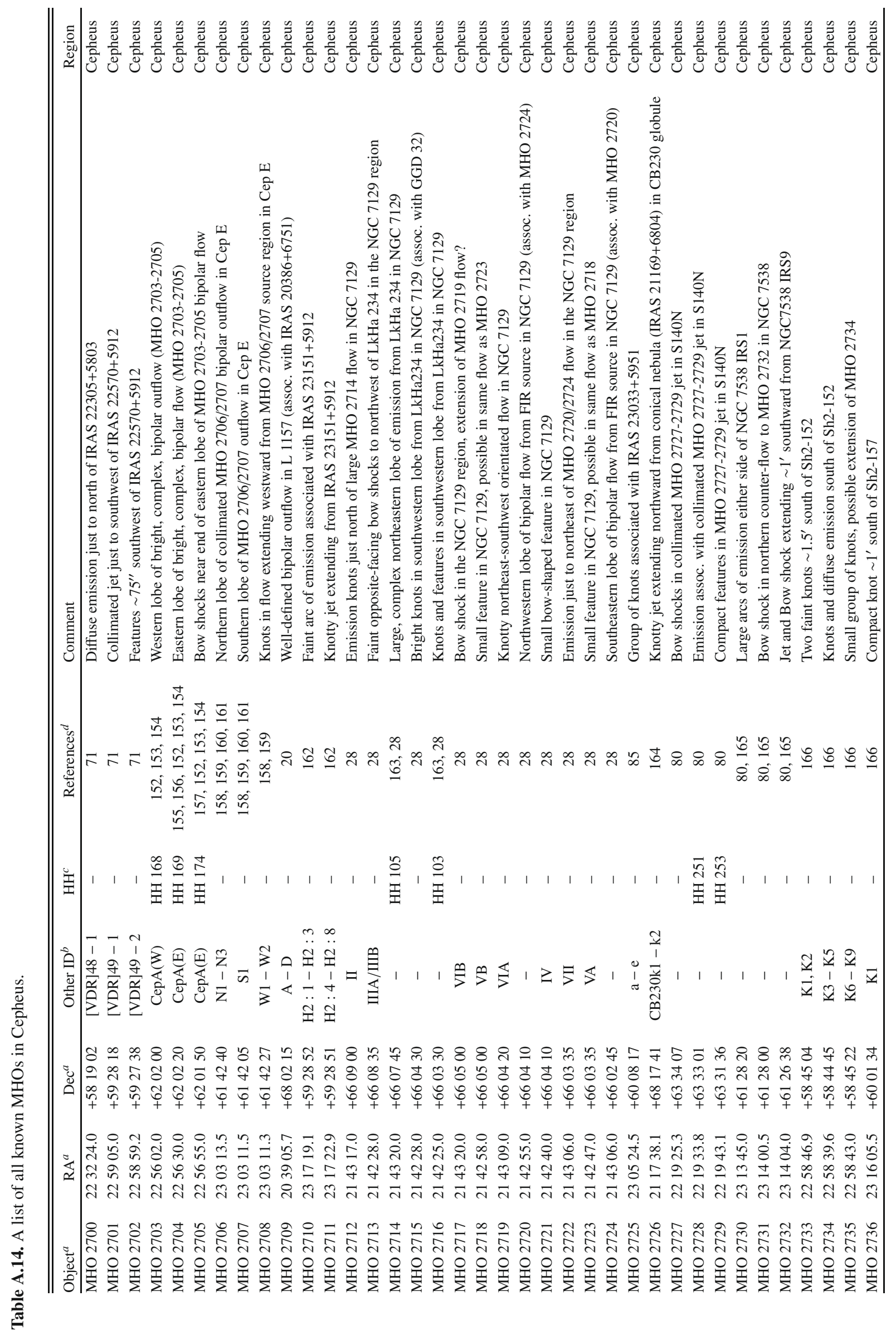


C. J. Davis et al.: A general catalogue of molecular hydrogen emission-line objects (MHOs) in outflows from young stars

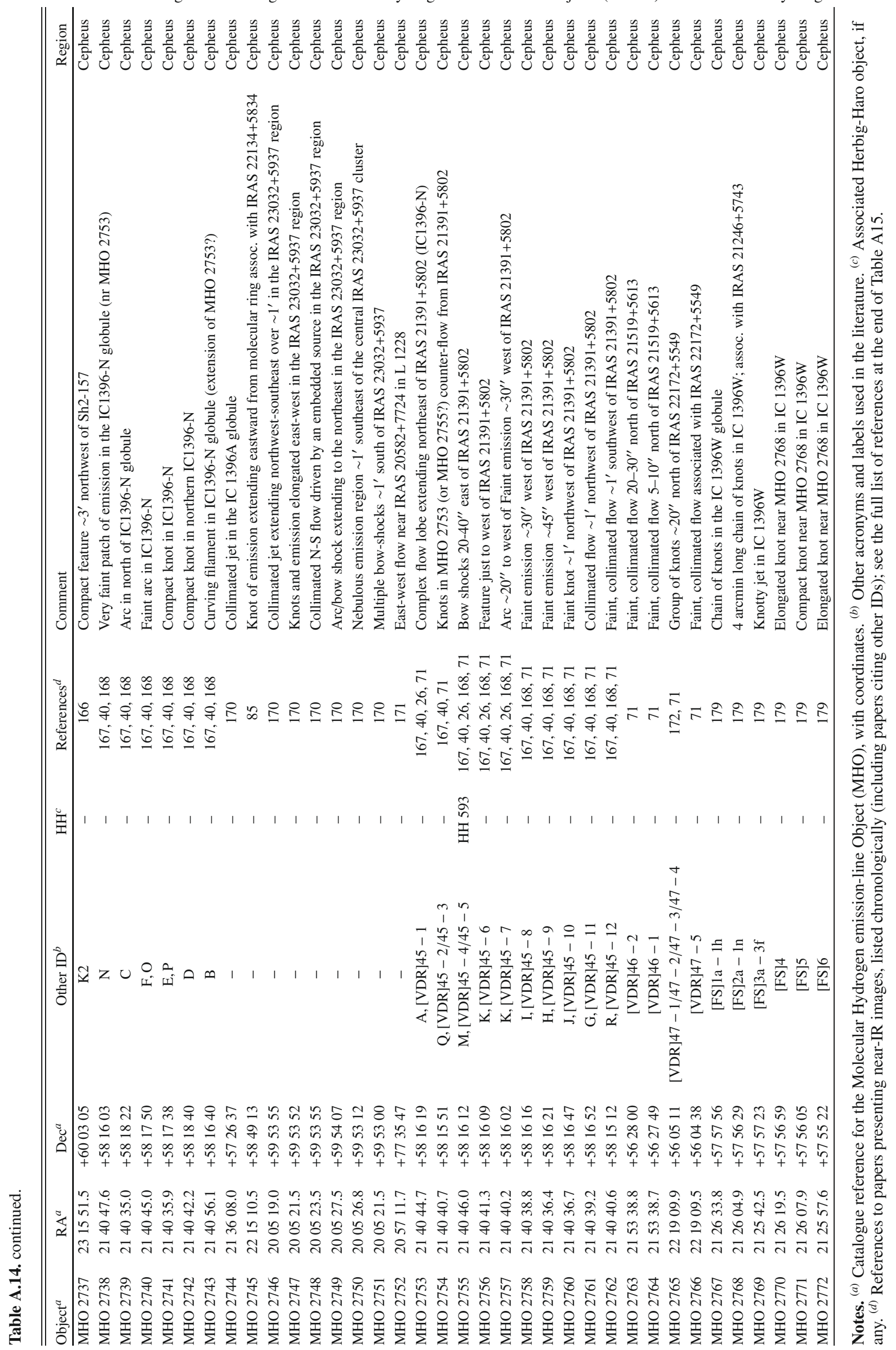




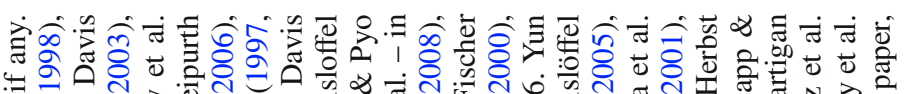

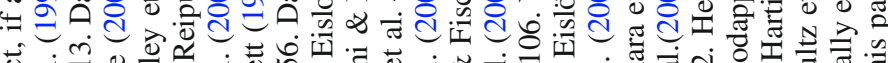

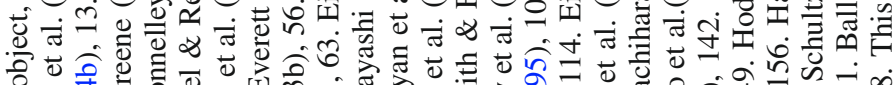

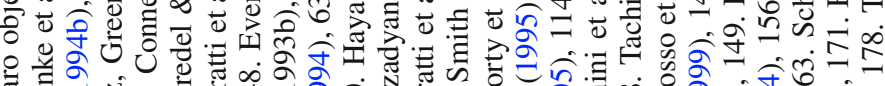

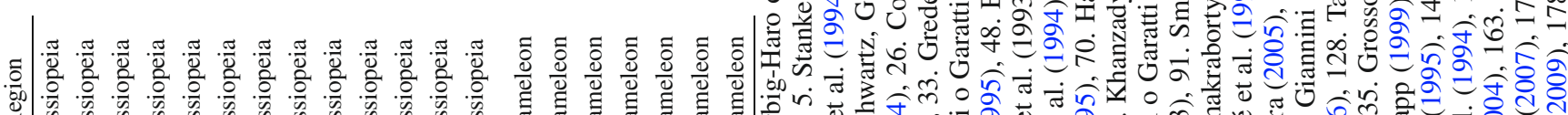
œ人

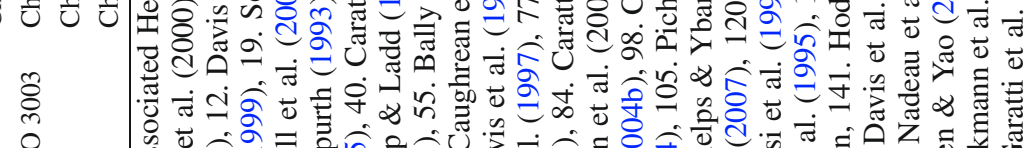
ơ

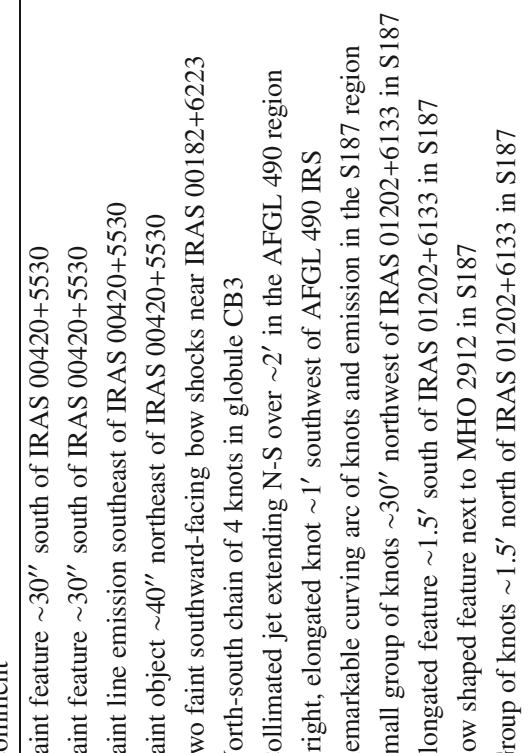
(n) 